\title{
Modeling and Analysis of Secondary Sources Coupling for Active Sound Field Reduction in Confined Spaces
}

\author{
Allahyar Montazeri ${ }^{*}$, C. James Taylor* \\ *Engineering Department, Lancaster University, Lancaster, UK, LA1 4YW. \\ 1a.montazeri@lancaster.ac.uk
}

\begin{abstract}
This article addresses the coupling of acoustic secondary sources in a confined space in a sound field reduction framework. By considering the coupling of sources in a rectangular enclosure, the set of coupled equations governing its acoustical behavior are solved. The model obtained in this way is used to analyze the behavior of multi-input multi-output (MIMO) active sound field control (ASC) systems, where the coupling of sources cannot be neglected. In particular, the article develops the analytical results to analyze the effect of coupling of an array of secondary sources on the sound pressure levels inside an enclosure, when an array of microphones is used to capture the acoustic characteristics of the enclosure. The results are supported by extensive numerical simulations showing how coupling of loudspeakers through acoustic modes of the enclosure will change the strength and hence the driving voltage signal applied to the secondary loudspeakers. The practical significance of this model is to provide a better insight on the performance of the sound reproduction/reduction systems in confined spaces when an array of loudspeakers and microphones are placed in a fraction of wavelength of the excitation signal to reduce/reproduce the sound field. This is of particular importance because the interaction of different sources affects their radiation impedance depending on the electromechanical properties of the loudspeakers.
\end{abstract}

Keywords: Active Sound Control System, Coupling of Sources, Enclosure, Loudspeaker and Microphone Array, Modal Analysis

\section{INTRODUCTION}

The problem of active sound field reduction/reproduction in enclosed spaces has been widely studied because of its practical significance. In case that the incident sound is annoying this problem is studied mostly under the title of active sound control in the literature. Nevertheless, when the sound field conveys some useful information it is desirable to control it actively while retaining some specific characteristics. Examples in which reduction of the target sound field in confined spaces is desired, include active control of tonal noise inside the cabin of aircraft and helicopters, i.e. noise resulting from blade passing frequency of the propeller driven aircraft and helicopters [1, 2]; and in cars at specific engine orders [18, 19, 22]. Fundamental theoretical and experimental investigations on proof of concept of active control techniques in the global reduction of harmonically excited enclosed sound fields are provided in references $[5,7,17]$. From the perspective of sound field reproduction active methods have shown promising results in applications such as immersive audio [25] and immersive communication systems [24].

Theoretical approaches of sound field reproduction systems in free field are referred to as Wave

Field Synthesis and Ambisonics [3]. However, inaccuracies in the reproduction due to the 
listener's movement and room reverberations led to the development of another technique known as multichannel inversion [4]. The underlying principles of this method share significant analogies with the theories of active control of sound fields [6]. The corresponding term associated with these two strategies in enclosed space are referred to as global and local control [8]. In global control, reduction of the acoustic pressure level at all points in the confined space is important (see e.g. [10] and the references therein) whilst local control aims to generate a quiet zone inside the enclosure (see e.g. [20,23] and the references therein). Two quantities used for global control of the sound field inside the enclosure are energy density and potential energy, whereas a norm of sound pressure at some discrete points in space is attempted most of the time to address local active noise control problems. Having a proper control strategy, the next important step in the design of an active control system is to find the optimal position for any secondary loudspeakers and error microphones according to the characteristics of the primary noise $[13,15]$. Most of the literature (e.g. $[11,17])$ suggests placement of secondary loudspeakers and error microphones at the corners of the enclosure to attenuate the low-frequency sound field in a global active control setting. However, analysis in reference [14] shows that when the dimensions of the enclosure are multiple integers of each other, placement of secondary loudspeakers at the corner of the cavity will not necessarily result in the maximum achievable global noise reduction.

Coupling of sources refers to the situation in which two coherent sources are placed within a fraction of the wavelength and affect each other's radiation impedance. This problem is studied in free space in reference [9]. In an enclosed space, the coupling happens through acoustic modes which in turn will change the radiation impedance of sources. However, relatively few articles to date have studied coupling of acoustic sources within a working environment $[12,21]$ and in the context of an active control system. A preliminary investigation by the present first author on modeling coupling of a loudspeaker with the acoustic modes in an enclosed space is reported in [16] where the results for two loudspeakers when no microphone is available are presented. In this article, we extend these results to an array of $L$ loudspeakers and $M$ microphones, and investigate the effect of coupling of secondary sources on the overall performance of an active sound control system in a confined space. The modeling and analysis performed in this paper explains some of the behaviors of active control systems occur in practice as a result of coupling of the secondary loudspeakers.

To be able to find a closed form solution for the problem, the enclosure is considered to have rigid boundary conditions. The proposed analytical model can be used to find an upper bound on the level of reduction of sound pressure and the required source strength when coupling exists 
among the sources in a multichannel active control system. The results are supported by extensive numerical simulations for both resonant and non-resonant frequencies of the enclosure. Preliminary results and notation are presented in section 2. A closed form solution and the associated theorems derived from modeling and analysis of the coupling of secondary sources in an ASC system with $L$ loudspeakers and $M$ microphones is given in section 3. These analytical results are obtained by generalization of the results from section 2 and are supported by extensive numerical simulations in section 5. Finally, the conclusions are presented in section 6

\section{PROBLEM FORMULATION AND STATEMENT}

\subsection{Notations and hypothesis}

In this section we present important definitions and notation used later in the article to formulate the problem. For all these definitions and assumptions refer to Fig. 1, where it is assumed that an array of $N$ loudspeakers is distributed inside the enclosure. For clarity, just two typical coupled loudspeakers, namely $\mathrm{SPK}_{1}$ and $\mathrm{SPK}_{2}$ are shown in Fig. 1. To make the formulation of the problem mathematically tractable, it is assumed that all loudspeakers inside the enclosure are in the $x z$ plane with different $y$ coordinates. Furthermore, the loudspeakers are considered to have square shapes of dimension $l$. To be more specific, the points on the surfaces of loudspeaker $\mathrm{SPK}_{\mathrm{i}}$ are defined by the set $\mathrm{SP}_{i}$ as follows:

$S P_{i}=\left\{(x, y, z) \mid y=y_{i}, x_{i} \leq x \leq x_{i}+l, z_{i} \leq z \leq z_{i}+l\right\}$

where $\left(x_{i}, y_{i}, z_{i}\right)$ is the corner point of the $i$ th loudspeaker. This is illustrated in Fig. 1 for $i=1,2$.

To be able to model coupling of loudspeakers they are considered to operate similar to a piston in response to the voltage signal applied to their terminal. The equation governing the sound pressure inside the enclosure when several loudspeakers are working together is stated in the frequency domain by the following standard partial differential equation:

$$
\frac{\omega^{2}}{c_{0}^{2}} p(r, \omega)+\nabla^{2} p(r, \omega)=-\rho_{0} i \omega u(r, \omega)
$$

where $r=(x, y, z)$ indicates a point in the Cartesian coordinate system and $c_{0}$ and $\rho_{0}$ are the velocity of sound and density of air respectively. In addition, $u(r, \omega)$ and $p(r, \omega)$ denote the total volume velocity and acoustic pressure inside the enclosure. By following the approach presented in [14], the solution of (2) with a rigid boundary condition assumption can be found 
using the separation principle. More specifically, the acoustic pressure inside the enclosure shown in Fig. 1 can be written as:

$$
p(r, \omega)=\sum_{n=1}^{\infty} a_{n}(\omega) \psi_{n}(r)
$$

where,

$\psi_{n}(r)=\sqrt{\varepsilon_{n_{1}} \varepsilon_{n_{2}} \varepsilon_{n_{3}}} \cos \left(\frac{n_{1} \pi x}{L_{1}}\right) \cos \left(\frac{n_{2} \pi y}{L_{2}}\right) \cos \left(\frac{n_{3} \pi z}{L_{3}}\right)$

is the shape of the $n$th acoustic mode of the enclosure and $\varepsilon_{v}$ is a coefficient used for normalization and is defined as:

$$
\varepsilon_{v}=\left\{\begin{array}{ll}
2 & \text { if } v>0 \\
1 & \text { if } v=0
\end{array} .\right.
$$

Moreover, $n_{1,}, n_{2}, n_{3}$ are integer indices representing the $n$th acoustic mode with amplitude $a_{n}(\omega)$ which is calculated as [14]:

$a_{n}(\omega)=\frac{\rho_{0} c_{0}^{2}}{V} A_{n}(\omega) \int_{\mathrm{V}} u(x, y, z, \omega) \psi_{n}(x, y, z) d x d y d z$

where,

$$
A_{n}(\omega)=\frac{i \omega}{-\omega^{2}+c_{0}^{2} \lambda_{n}^{2}+i \omega \zeta_{n} c_{0}^{2} \lambda_{n}^{2}}
$$

and $\zeta_{n}$ and $\lambda_{n}$ are the damping and natural frequency of the $n$th acoustic mode of enclosure. In equation (7), $\psi_{n}(x, y, z)$ represents the shape of the $n$th acoustic mode and integration is performed over the entire volume $V$ of the enclosure.

The net acoustic pressure on the surface of each loudspeaker is calculated by taking the average of pointwise acoustic pressure on the surface of each loudspeaker. This is obtained for the $i$ th loudspeaker as follows

$$
P_{i}(\omega)=\frac{1}{S_{i}} \int_{\mathrm{SP}_{i}} p\left(x, y_{i}, z, \omega\right) \mathrm{d} s
$$

The total volume velocity (in $\mathrm{m}^{3} / \mathrm{s}$ ) of the $i$ th loudspeaker is achieved by integrating the volume velocity per unit surface, of all points on the surface of the loudspeakers, i.e.,

$U_{i}(\omega)=\int_{\mathrm{SP}_{i}} u\left(x, y_{i}, z, \omega\right) \mathrm{d} s$ 


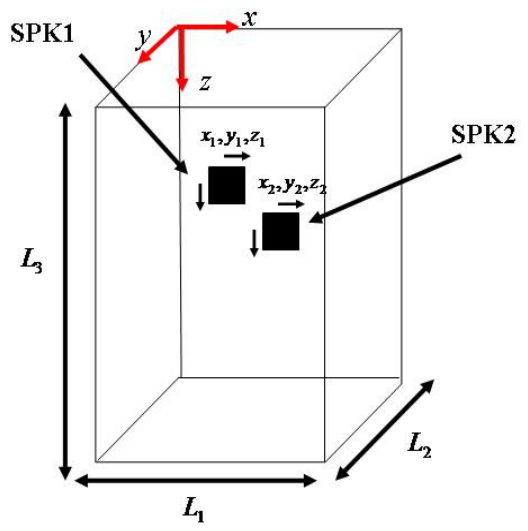

Figure 1. A typical rectangular enclosure with coupled secondary loudspeakers.

The pointwise volume velocity on the surface of the $i$ th loudspeaker with a uniform distribution hypothesis is expressed as:

$u_{i}\left(x, y_{i}, z, \omega\right)=\frac{U_{i}(\omega)}{S_{i}}\left[H\left(x-x_{i}\right)-H\left(x-x_{i}-l\right)\right]\left[H\left(z-z_{i}\right)-H\left(z-z_{i}-l\right)\right]$

where $S_{i}$ is the surface area of loudspeaker $\mathrm{SPK}_{\mathrm{i}}$. It is possible to relate the total volume velocity on the surface of the $i$ th loudspeaker to the movement of its coils using the following integral

$S_{i} \dot{x}_{i}(t)=\int_{\mathrm{S}_{i}} u\left(x, y_{i}, z, t\right) \mathrm{d} s$

where $x_{i}(t)$ is the displacement of the coil and diaphragm of $\mathrm{SPK}_{\mathrm{i}}$.

In the case that $L$ loudspeakers are distributed inside the enclosure, using the principle of superposition, the volume velocity $u(x, y, z, \omega)$ in equation (7) can be written as

$u(x, y, z, \omega)=\sum_{l=1}^{L} u_{i}\left(x, y_{i}, z, \omega\right)$

where $u_{i}\left(x, y_{i}, z, \omega\right)$ is defined by equation (10). Substituting $u(x, y, z, \omega)$ from equation (9) into (7) will determine the acoustic pressure in the modal space,

$a_{n}(\omega)=\frac{\rho_{0} c_{0}^{2}}{V} A_{n}(\omega) \frac{1}{S} \sum_{l=1}^{L} U_{l}(\omega) \int_{\mathrm{SP}_{1}} \psi_{n}\left(x, y_{l}, z\right) \mathrm{d} x \mathrm{~d} z$

Here it is assumed that all loudspeakers are identical with the same area equal to $S$.

\subsection{Preliminary results}

The first step towards modeling coupling of loudspeakers inside the enclosure is to find out how the voltage applied across the input terminals of a loudspeaker will result in the required volume 
Table 1: List of parameters

\begin{tabular}{|c|c|}
\hline Symbol & Definition of parameter \\
\hline$R_{i}$ & Resistance of the loudspeaker coil \\
\hline$L_{i}$ & Inductance of the loudspeaker coil \\
\hline$B_{i}$ & Permanent magnetic field of the loudspeaker \\
\hline$l_{i}$ & Length of voice coil in the magnetic field \\
\hline$m_{i}$ & Mass of loudspeaker \\
\hline$c_{i}$ & Damping of loudspeaker \\
\hline$k_{i}$ & Stiffness of loudspeaker \\
\hline
\end{tabular}

velocity and acoustic pressure on the surface of that loudspeaker. We assume that an array of $L$ loudspeakers is distributed inside the enclosure, as shown in Fig. 1, and each loudspeaker operates at low frequencies as a piston. In this case, it is possible to model each loudspeaker as a simple mass-spring-damper system. By following the approach presented in reference [16], for each loudspeaker $\mathrm{SPK}_{\mathrm{i}}$, the equation that relates the input voltage applied to the loudspeaker to the volume velocity and acoustic pressure produced on the surface of loudspeaker $\mathrm{SPK}_{\mathrm{i}}$, can be written in the frequency domain as ,

$Z_{\mathrm{S}_{i}}(\omega) U_{i}(\omega)+S_{i} P_{i}(\omega)=Z_{i}(\omega) V_{\mathrm{e} i}(\omega)$

where $V_{\mathrm{e} i}(\omega)$ is the voltage applied to the loudspeaker $i$ and

$U_{i}(\omega)=S_{i} j \omega X_{i}(\omega)$

$Z_{\mathrm{S}_{i}}(\omega)=\frac{1}{j \omega S_{i}}\left[-m_{i} \omega^{2}+\left(c_{i}+\frac{\left(B_{i} l_{i}\right)^{2}}{R_{i}+j L_{i} \omega}\right) j \omega+k_{i}\right]$

$Z_{i}(\omega)=\frac{B_{i} l_{i}}{R_{i}+j L_{i} \omega}$

The list of all parameters in equations (9) to (11) along with their definitions are presented in Table 1. The detailed derivation of equations (9) to (11) is presented in [16] and to avoid duplication it is not repeated here. The most important indication of relation (9), is that the volume velocity $U_{i}(\omega)$ is determined as a result of the voltage applied to the terminals of the loudspeaker and the acoustic pressure on the surface of the loudspeaker as a result of coupling with other loudspeakers through acoustic modes of the enclosure. This is not the case in almost all modeling work to date, in which the loudspeaker is generally assumed to be rigid and hence 
$P_{i}(\omega)$ will be equal to zero. Therefore, in case of lack of coupling, equation (9) will be simplified as follows:

$U_{i}(\omega)=\frac{Z_{i}(\omega) V_{\mathrm{e} i}(\omega)}{Z_{S_{i}}(\omega)}$

where $U_{i}(\omega)$ is a sole linear function of $V_{\mathrm{e} i}(\omega)$. This reveals that coupling of the acoustic sources with the environment inside the enclosure changes the amplitude and phase of the source strength, and consequently the pattern of acoustic pressure inside the enclosure. However, it can also be inferred from equation (9) that even when zero voltage is applied to the terminal of loudspeaker $\mathrm{SPK}_{\mathrm{i}}$, the source strength depends on the net external acoustic pressure applied to the surface of $\mathrm{SPK}_{\mathrm{i}}$ from the surrounding environment. To be able to derive $U_{i}(\omega)$ as a direct function of $V_{\mathrm{e} i}(\omega)$ in (9), it is necessary to substitute for $P_{i}(\omega)$ in terms of $U_{i}(\omega)$. These results for two loudspeakers are presented in reference [16]. However, for the sake of clarity on how to derive the results for $L$ loudspeakers, it is repeated here for a single loudspeaker. From equation (13), the acoustic pressure in the modal space for $L=1$ is,

$a_{n}(\omega)=\frac{\rho_{0} c_{0}^{2}}{V} A_{n}(\omega) \frac{U_{1}(\omega)}{S_{1}} \int_{\mathrm{SP}_{1}} \psi_{n}\left(x, y_{1}, z\right) \mathrm{d} x \mathrm{~d} z$

Substituting $a_{n}(\omega)$ from equation (18) into equation (3) and then integrating the achieved sound pressure over the surface of the loudspeaker SPK1 from equation (8) yields,

$$
S_{1} P_{1}(\omega)=\frac{\rho_{0} c_{0}^{2}}{V S_{1}} U_{1}(\omega) \sum_{n=0}^{\infty} A_{n}(\omega)\left(\int_{\mathrm{SP}_{1}} \psi_{n}\left(x, y_{1}, z\right) \mathrm{d} s\right)^{2}
$$

Substitution of $P_{1}(\omega)$ from (19) into (14) and then factorizing $U_{1}(\omega)$ results in,

$$
U_{1}(\omega)=\frac{Z_{1}(\omega) V_{\mathrm{el}}(\omega)}{Z_{S_{1}}(\omega)+\frac{\rho_{0} c_{0}^{2}}{V S} \sum_{n=0}^{\infty} A_{n}(\omega)\left(\int_{\mathrm{SP}_{1}} \psi_{n}\left(x, y_{1}, z\right) \mathrm{d} s\right)^{2}}
$$

As we have only a single loudspeaker in this preliminary analysis, the coupling is happening with the acoustic modes of the enclosure. However, in the case that multiple acoustic sources exist inside the enclosure, each loudspeaker affects the acoustic modes of the enclosure in turn and the result after superposition will appear as the net acoustic pressure on the surface of all loudspeakers in the form of coupling. A novel generalization of these results for an array of $L$ loudspeakers and $M$ microphones is developed in the next section. 


\section{GENERALIZATION TO MULTI-CHANNEL ASC SYSTEM}

\subsection{Coupling of an array of L loudspeakers}

In the first instance, it is assumed that an array of $L$ identical loudspeakers, i.e. $\mathrm{SPK}_{1}, \mathrm{SPK}_{2}, \ldots$, $\mathrm{SPK}_{\mathrm{L}}$, are operating as secondary sources for an ASC system inside the enclosure shown in Fig.1. Without loss of generality we assume that all physical parameters of the loudspeakers listed in Table 1 are the same and no microphone exists inside the enclosure. When coupling exists among an array of $L$ loudspeakers, the total volume velocity of each loudspeaker is a function of all voltages applied to other secondary sources, and hence the derived expression will be more complicated than the result given in (20). To be able to find a closed form solution for the volume velocity of each loudspeaker $U_{i}(\omega)$ for $i=1,2, \cdots L$ we start from (13). Substitution of $a_{n}(\omega)$ from equation (13) into (3) will determine the acoustic pressure inside the enclosure as a function of volume velocity of all $L$ loudspeakers. By introducing the resulting expression into (8) it is possible to find the net acoustic pressure applied on the surface of each loudspeaker. For example, the expression for $\mathrm{SPK}_{1}$ becomes:

$$
S P_{1}(\omega)=\frac{\rho_{0} c_{0}^{2}}{V S} \sum_{n=0}^{\infty} A_{n}(\omega)\left(\sum_{l=1}^{L} U_{l}(\omega) \int_{\mathrm{SP}_{1}} \psi_{n}\left(x, y_{l}, z\right) \mathrm{d} x \mathrm{~d} z\right) \int_{\mathrm{SP}_{1}} \psi_{n}\left(x, y_{1}, z\right) \mathrm{d} x \mathrm{~d} z
$$

Changing the order of summations yields,

$$
S P_{1}(\omega)=\frac{\rho_{0} c_{0}^{2}}{V S} \sum_{l=1}^{L} U_{l}(\omega) \sum_{n=0}^{\infty} A_{n}(\omega)\left(\int_{\mathrm{SP}_{1}} \psi_{n}\left(x, y_{l}, z\right) \mathrm{d} x \mathrm{~d} z\right)\left(\int_{\mathrm{SP}_{1}} \psi_{n}\left(x, y_{1}, z\right) \mathrm{d} x \mathrm{~d} z\right)
$$

Using the same approach, the net acoustic pressure on the surface of $\mathrm{SPK}_{2}$ is calculated as follows:

$$
S P_{2}(\omega)=\frac{\rho_{0} c_{0}^{2}}{V S} \sum_{l=1}^{L} U_{l}(\omega) \sum_{n=0}^{\infty} A_{n}(\omega)\left(\int_{\mathrm{SP}_{1}} \psi_{n}\left(x, y_{l}, z\right) \mathrm{d} x \mathrm{~d} z\right)\left(\int_{\mathrm{SP}_{2}} \psi_{n}\left(x, y_{2}, z\right) \mathrm{d} x \mathrm{~d} z\right)
$$

By following the same principle, it is possible to find the acoustic pressure on the surface of all $L$ loudspeakers. Putting the set of equations relating the volume velocities of the $L$ sources to their net acoustic pressure in matrix form yields:

$$
\left[\begin{array}{c}
S P_{1}(\omega) \\
S P_{2}(\omega) \\
\vdots \\
S P_{L}(\omega)
\end{array}\right]=\mathbf{Z}_{c}(\omega)\left[\begin{array}{c}
U_{1}(\omega) \\
U_{2}(\omega) \\
\vdots \\
U_{L}(\omega)
\end{array}\right]
$$

where $\mathbf{Z}_{c}(\omega)$ is an $L \times L$ symmetric coupling matrix whose $i j$ th element is written as: 
$\left[\mathbf{Z}_{c}(\omega)\right]_{i j}=\frac{\rho_{0} c_{0}^{2}}{V S} \sum_{n=0}^{\infty} A_{n}(\omega)\left(\int_{\mathrm{SP}_{\mathrm{i}}} \psi_{n}\left(x, y_{i}, z\right) \mathrm{d} x \mathrm{~d} z\right)\left(\int_{\mathrm{SP}_{\mathrm{j}}} \psi_{n}\left(x, y_{j}, z\right) \mathrm{d} x \mathrm{~d} z\right)$

Writing equation (14) for all $L$ loudspeakers $\mathrm{SPK}_{1}, \mathrm{SPK}_{2}, \ldots, \mathrm{SPK}_{\mathrm{L}}$ and formulating this in matrix form yields:

$Z_{s}(\omega)\left[\begin{array}{c}U_{1}(\omega) \\ U_{2}(\omega) \\ \vdots \\ U_{L}(\omega)\end{array}\right]+\left[\begin{array}{c}S P_{1}(\omega) \\ S P_{2}(\omega) \\ \vdots \\ S P_{L}(\omega)\end{array}\right]=Z(\omega)\left[\begin{array}{c}V_{e 1}(\omega) \\ V_{e 2}(\omega) \\ \vdots \\ V_{e L}(\omega)\end{array}\right]$

Substitution of the vector of net acoustic pressure sources from equation (24) into (26) makes it possible to derive the total volume velocity of all $L$ secondary sources as a function of the voltages applied to all sources in a linear but coupled fashion, as follows:

$\left[\begin{array}{c}U_{1}(\omega) \\ U_{2}(\omega) \\ \vdots \\ U_{L}(\omega)\end{array}\right]=\overline{\mathbf{Z}}_{c}(\omega)\left[\begin{array}{c}V_{e 1}(\omega) \\ V_{e 2}(\omega) \\ \vdots \\ V_{e L}(\omega)\end{array}\right]$

$\overline{\mathbf{Z}}_{c}(\omega)=\left(Z_{s}(\omega) I+\mathbf{Z}_{c}(\omega)\right)^{-1} Z(\omega)$

By definition of a proper performance index in terms of volume velocity of acoustic sources and then replacing these in terms of $V_{e 1}(\omega), V_{e 2}(\omega), \ldots V_{e L}(\omega)$ from equation (27), it would be possible to control the sound field inside the enclosure by considering coupling of secondary loudspeakers. As can be seen from equation (27) the coupling of secondary sources will change the strength of sources through the matrix $\overline{\mathbf{Z}}_{c}(\omega)$.

Lemma 1. For any specific frequency $\omega$ the complex $L \times L$ matrix $\mathbf{Z}_{c}(\omega)$ is symmetric positive (or negative) definite.

Proof: By looking at the $i j$ th element of the matrix $\mathbf{Z}_{c}(\omega)$ from (25) it can be clearly seen that $\left[\mathbf{Z}_{c}(\omega)\right]_{i j}=\left[\mathbf{Z}_{c}(\omega)\right]_{j i}$ and hence $\mathbf{Z}_{c}(\omega)$ is symmetric. The positive definiteness of the complex matrix $\mathbf{Z}_{c}(\omega)$ for $L=1,2$ is proved in reference [16]. For $L \geq 2$ we prove the lemma by induction. It is well-known that a complex matrix is positive definite if and only if its real part is positive definite. The real part of $\mathbf{Z}_{c}(\omega)$ is obtained by taking the real part of $A_{n}(\omega)$ in equation (25). Assuming that $\operatorname{Re}\left[\mathbf{Z}_{c}(\omega)\right]$ is an $L \times L$ positive definite matrix we need to prove that this remains true when $L+1$ loudspeakers are placed inside the enclosure. By definition of positive definiteness for the $L \times L$ matrix $\operatorname{Re}\left[\mathbf{Z}_{c}(\omega)\right]$, it can be written,

$\mathbf{x}^{T} \operatorname{Re}\left[\mathbf{Z}_{c}(\omega)\right] \mathbf{x} \geq 0 \quad \forall \mathbf{x} \neq 0$ 
where $\mathbf{x}$ is an arbitrary $L \times 1$ vector defined as $\mathbf{x}=\left[x_{1} \ldots \ldots x_{L}\right]^{T}$. Writing (29) in terms of the elements of the matrix $\operatorname{Re}\left[\mathbf{Z}_{c}(\omega)\right]$ yields,

$$
\frac{\rho_{0} c_{0}^{2}}{V S} \sum_{i=1}^{L} \sum_{j=1}^{L} \sum_{n=0}^{\infty} x_{i} x_{j} \operatorname{Re}\left[A_{n}(\omega)\right]\left(\int_{\mathrm{SP}_{\mathrm{i}}} \psi_{n}\left(x, y_{i}, z\right) \mathrm{d} x \mathrm{~d} z\right)\left(\int_{\mathrm{SP}_{\mathrm{j}}} \psi_{n}\left(x, y_{j}, z\right) \mathrm{d} x \mathrm{~d} z\right) \geq 0
$$

By defining,

$\alpha_{n i}=\sqrt{\frac{\rho_{0} c_{0}^{2}}{V S}} x_{i}\left(\int_{\mathrm{SP}_{\mathrm{i}}} \psi_{n}\left(x, y_{i}, z\right) \mathrm{d} x \mathrm{~d} z\right)$

equation (30) can be rewritten as,

$\sum_{i=1}^{L} \sum_{j=1}^{L} \sum_{n=0}^{\infty} \alpha_{n i} \alpha_{n j} \operatorname{Re}\left[A_{n}(\omega)\right] \geq 0$

Changing the order of summations in (32) yields,

$\sum_{n=0}^{\infty} \operatorname{Re}\left[A_{n}(\omega)\right] \sum_{i=1}^{L} \sum_{j=1}^{L} \alpha_{n i} \alpha_{n j} \geq 0$

The inner summation in (33) can be written as a non-negative coefficient as follows,

$\beta_{n}=\sum_{i=1}^{L} \sum_{j=1}^{L} \alpha_{n i} \alpha_{n j}=\left(\sum_{i=1}^{L} \alpha_{n i}\right)^{2} \geq 0$

By combining (33) and (34) the hypothesis of induction will be written as,

$$
\sum_{n=0}^{\infty} \beta_{n} \operatorname{Re}\left[A_{n}(\omega)\right] \geq 0 \quad \forall \beta_{n} \geq 0
$$

Finally, by adding one more loudspeaker and repeating equations (30) to (34) we achieve the same results with a new set of coefficients $\beta_{n}^{\prime}$ which are calculated as follows:

$\beta_{n}^{\prime}=\sum_{i=1}^{L+1} \sum_{j=1}^{L+1} \alpha_{n i} \alpha_{n j}=\left(\sum_{i=1}^{L+1} \alpha_{n i}\right)^{2} \geq 0$

Therefore, since $\beta_{n}^{\prime} \geq 0$ and using the hypothesis of induction (34) it can be readily proven that,

$\sum_{n=0}^{\infty} \beta_{n}^{\prime} \operatorname{Re}\left[A_{n}(\omega)\right] \geq 0$

This completes the proof of Lemma $1 \bullet$.

Remark 1. From (25) it can be inferred that the diagonal elements of $\mathbf{Z}_{c}(\omega)$ concern the selfcoupling of each loudspeaker with the acoustic modes of the enclosure, while the off-diagonal elements concern the cross-coupling between each pair of sources. In the case that coupling between sources does not exist, namely $\mathbf{Z}_{c}(\omega)=0$, it can be deduced from (27) and (28) that the volume velocity of each loudspeaker merely depends on the input voltage of that loudspeaker 
through the diagonal matrix $\overline{\mathbf{Z}}_{c}(\omega)$. By contrast, the off-diagonal zeros in this matrix imply zero coupling between sources.

Remark 2. Noting the structure of the matrix $\mathbf{Z}_{c}(\omega)$ in (25), it can be seen that this matrix will lose rank only at a few distinct frequencies, i.e. zeros of the frequency response of the system in the specified bandwidth, or when the coupled loudspeakers are placed in a specific way such that the rows of the matrix $\mathbf{Z}_{c}(\omega)$ becomes linearly dependent at all frequencies. A trivial example is the case that all loudspeakers are placed in one position. In such cases the minimum singular value of $\mathbf{Z}_{c}(\omega)$, i.e. $\underline{\sigma}_{c}$, tends to zero. Therefore, we say the ASC system is in a singular configuration when the matrix $\mathbf{Z}_{c}(\omega)$ becomes singular.

Remark 3. Without loss of generality it is assumed that all loudspeakers are identical with similar physical properties. Hence, for all loudspeakers, $Z_{\mathrm{S}_{i}}(\omega)$ and $Z_{i}(\omega)$ are considered to be equal to $Z_{s}(\omega)$ and $Z(\omega)$ respectively.

Remark 4. As a general notation and to distinguish the variables indicating the coupling of loudspeakers in the model with those when coupling is ignored, we use a dashed line on top of the variables.

\subsection{Formulation of coupling for an active noise control system}

In this section, we formulate the effect of coupling of secondary sources in case that an array of loudspeakers and microphones are placed inside the enclosure. Since the primary noise most of the time is generated by another source, it is normal to assume that it is independent with respect to the secondary loudspeakers. Here we assume the primary noise is generated by a loudspeaker placed in the corner of the enclosure as shown in Fig. 1 and that no coupling exists for this source. Considering the effect of primary and secondary sources, the acoustic pressure inside the enclosure in a modal space can be calculated using the principle of superposition:

$a_{n}(\omega)=a_{n \mathrm{p}}(\omega)+\sum_{l=1}^{L} a_{n l}(\omega)$

where $a_{n \mathrm{p}}(\omega)$ is the modal amplitude of sound pressure resulting from the primary source, and $a_{n l}(\omega)$ for $l=1,2, \ldots L$ is the modal amplitude of the sound pressure resulting from the effect of the secondary loudspeakers $\mathrm{SPK}_{1}, \mathrm{SPK}_{2}, \ldots, \mathrm{SPK}_{\mathrm{n}}$. Substituting $U_{l}(\omega)$ for $l=1,2, \ldots, L$ from the matrix form (27) into equation (13) yields the modal amplitude of the sound pressure produced due to each individual secondary source as follows: 
$a_{n l}(\omega)=\frac{\rho_{0} c_{0}^{2}}{V S} A_{n}(\omega) \sum_{k=1}^{L} \bar{z}_{l k}(\omega) V_{e k}(\omega) \int_{\mathrm{SP}_{l}} \psi_{n}\left(x, y_{l}, z\right) \mathrm{d} x \mathrm{~d} z$,

where $\bar{z}_{l k}(\omega)$ is the $l k$ th component of the matrix $\overline{\mathbf{Z}}(\omega)$ expressed in equation (28). By combining the result obtained from the expression in (38) with equation (37), it is possible to derive a closed form for the acoustic pressure in modal space as a function of the control input voltages applied to the secondary sources as follows:

$\sum_{l=1}^{L} p_{n l}(\omega)=\frac{\rho_{0} c_{0}^{2}}{V S} A_{n}(\omega) \sum_{l=1}^{L} \sum_{k=1}^{L} \bar{z}_{l k}(\omega) V_{e k}(\omega) \int_{\mathrm{SP}_{l}} \psi_{n}\left(x, y_{l}, z\right) \mathrm{d} x \mathrm{~d} z$

Changing the order of summations in equation (39) yields:

$\sum_{l=1}^{L} p_{n l}(\omega)=\frac{\rho_{0} c_{0}^{2}}{V S} A_{n}(\omega) \sum_{k=1}^{L}\left[\sum_{l=1}^{L} \bar{z}_{l k}(\omega) \int_{\mathrm{SP}_{l}} \psi_{n}\left(x, y_{l}, z\right) \mathrm{d} x \mathrm{~d} z\right] V_{e k}(\omega)$.

By defining $\bar{b}_{n k}(\omega)$ as:

$\bar{b}_{n k}(\omega)=\frac{\rho_{0} c_{0}^{2}}{V S} A_{n}(\omega) \sum_{l=1}^{L} \bar{z}_{l k}(\omega) \int_{\mathrm{SP}_{l}} \psi_{n}\left(x, y_{l}, z\right) \mathrm{d} x \mathrm{~d} z$

equation (37) is written as:

$a_{n}(\omega)=a_{n \mathrm{p}}(\omega)+\sum_{k=1}^{L} \bar{b}_{n k}(\omega) V_{e k}(\omega)$

If we limit the bandwidth of the controller and hence the active control system to the first $N$ acoustic modes of the enclosure, it would be possible to write expression (42) in the matrix form as:

$\mathbf{a}=\mathbf{a}_{\mathrm{p}}+\overline{\mathbf{B}} \mathbf{v}_{\mathrm{e}}$

Here $\mathbf{a}$ and $\mathbf{a}_{\mathrm{p}}$ are the vectors of complex modal amplitudes due to the effect of primary and secondary loudspeakers and $\mathbf{v}_{\mathrm{e}}$ is the vector of voltages applied to the array of secondary loudspeakers. The index $\omega$ is omitted for simplicity and $\overline{\mathbf{B}}$ is an $N \times L$ matrix whose components are defined by equation (41). Rewriting (3) in matrix form and combining it with (43) make it possible to find the acoustic pressure at different points inside the enclosure, as follows:

$\mathbf{p}=\boldsymbol{\Psi}^{T} \mathbf{a}$ 
where $\mathbf{p}$ is a $M \times 1$ vector showing acoustic pressure at $M$ discrete points inside the enclosure and $\boldsymbol{\Psi}$ is an $N \times M$ matrix whose elements $(n, m)$ are the value of the shape of the $n^{\text {th }}$ mode at point $m$.

A suitable performance index to formulate the active noise control system in case of coupling is acoustic potential energy inside the enclosure and is calculated by

$E_{\mathrm{P}}=\frac{V}{4 \rho_{0} c_{0}^{2}} \mathbf{a}^{H} \mathbf{a}$

By minimizing $E_{\mathrm{P}}$ in equation (45), the optimal excitation voltage of the secondary loudspeakers as well as the maximum acoustic potential energy reduction inside enclosure is [14]

$\overline{\mathbf{v}}_{\mathrm{eo}}=-\left[\overline{\mathbf{B}}^{H} \overline{\mathbf{B}}\right]^{-1} \overline{\mathbf{B}}^{H} \mathbf{a}_{\mathrm{p}}$

$\frac{E_{\mathrm{po}}}{E_{\mathrm{pp}}}=1-\frac{\mathbf{a}_{\mathrm{p}}{ }^{H} \overline{\mathbf{B}}\left[\overline{\mathbf{B}}^{H} \overline{\mathbf{B}}\right]^{-1} \overline{\mathbf{B}}^{H} \mathbf{a}_{\mathrm{p}}}{\mathbf{a}_{\mathrm{p}}{ }^{H} \mathbf{a}_{\mathrm{p}}}$

Here $\overline{\mathbf{v}}_{\text {eо }}$ is used to distinguish the optimal excitation voltage in case of coupling with $\mathbf{v}_{\text {eo }}$ when no coupling exists. The latter corresponds to the case where the matrix $\overline{\mathbf{B}}$ in (46) and (47) is replaced with $\mathbf{B}$. The simplest way to approximate this quantity in practice is to deploy an array of $M$ microphones at proper positions inside the enclosure. In this case, the acoustic potential energy of the enclosure is estimated by:

$J_{\mathrm{P}}=\frac{V}{4 \rho_{0} c_{0}{ }^{2} M} \mathbf{p}^{H} \mathbf{p}$

where $\mathbf{p}$ is defined by (44). Combining (43) and (44) and replacing $\mathbf{p}$ in equation (48) with that from equation (44) yields:

$\mathbf{p}=\boldsymbol{\Psi}^{T} \mathbf{a}_{\mathrm{p}}+\boldsymbol{\Psi}^{T} \overline{\mathbf{B}} \mathbf{v}_{\mathrm{e}}=\mathbf{p}_{\mathrm{p}}+\overline{\mathbf{G}} \mathbf{v}_{\mathrm{e}}$

$\overline{\mathbf{G}}=\boldsymbol{\Psi}^{T} \overline{\mathbf{B}}$

where $\overline{\mathbf{G}}$ is an $M \times L$ matrix of frequency response functions from secondary sources to error microphones when coupling exists between sources, and $\mathbf{p}_{\mathrm{p}}$ is the acoustic pressure sensed by the microphones as a result of primary noise. Applying $\mathbf{p}$ from equation (49) to equation (48) and minimizing the resulting quadratic performance index yields the optimal excitation voltage of the secondary loudspeakers in case of coupling among secondary sources:

$\overline{\mathbf{v}}_{\text {so }}=-\left[\overline{\mathbf{G}}^{H} \overline{\mathbf{G}}\right]^{-1} \overline{\mathbf{G}}^{H} \mathbf{p}_{\mathrm{p}}$ 


$$
\frac{J_{\mathrm{po}}}{J_{\mathrm{pp}}}=1-\frac{\mathbf{p}_{\mathrm{p}}{ }^{H} \overline{\mathbf{G}}\left[\overline{\mathbf{G}}^{H} \overline{\mathbf{G}}\right]^{-1} \overline{\mathbf{G}}^{H} \mathbf{p}_{\mathrm{p}}}{\mathbf{p}_{\mathrm{p}}{ }^{H} \mathbf{p}_{\mathrm{p}}}
$$

Here $\overline{\mathbf{v}}_{\text {so }}$ is used to represent the optimal excitation voltage in case of coupling and when microphones are present. To compare the results with the case that no coupling exists among sources, it is necessary to find a relation between the frequency response functions $\overline{\mathbf{G}}$ and $\mathbf{G}$. According to (50) this problem is equivalent to finding the relation between the matrices $\overline{\mathbf{B}}$ and B . This problem is addressed in the following sections of the article using both analytical and numerical results.

\subsection{Comparison with the uncoupled case}

To answer the question of how coupling of secondary loudspeakers will influence the behavior of a multichannel active noise control system in a confined space, it is necessary to find the relationship between the matrices $\overline{\mathbf{B}}$ and $\mathbf{B}$ for the coupled and uncoupled sources respectively. We can extract elements of the matrix $\mathbf{B}$, i.e. $b_{n k}(\omega)$, by referring to reference [14]:

$b_{n k}(\omega)=\frac{\rho_{0} c_{0}^{2}}{V S} A_{n}(\omega) \frac{Z(\omega)}{Z_{s}(\omega)} \int_{\mathrm{SP}_{k}} \psi_{n}\left(x, y_{k}, z\right) \mathrm{d} x \mathrm{~d} z$

Comparing these elements with those of $\overline{\mathbf{B}}$ derived in equation (41) demonstrates that $\bar{b}_{n k}(\omega)$ can be expressed in terms of $b_{n k}(\omega)$ as follows:

$\bar{b}_{n k}(\omega)=\frac{Z_{s}(\omega)}{Z(\omega)} \sum_{l=1}^{L} \bar{z}_{l k}(\omega) b_{n l}(\omega)$

Writing equation (54) in matrix form:

$\overline{\mathbf{B}}=\mathbf{B} \overline{\mathbf{Z}}_{c}$

Here, for the sake of simplicity, the index $\omega$ is omitted from both sides and $\overline{\mathbf{Z}}_{c}(\omega)$ is defined as:

$\overline{\mathbf{Z}}_{c}(\omega)=\left(Z_{s}(\omega) \mathbf{I}+\mathbf{Z}_{c}(\omega)\right)^{-1} Z_{s}(\omega)$

Using the relationship between the matrices $\overline{\mathbf{B}}$ and $\mathbf{B}$ it is possible to find the relationship between the matrix of frequency response functions between loudspeakers and microphones, as shown by the following lemma.

Lemma 2. Assume $L$ loudspeakers and $M$ microphones are distributed inside the enclosure shown in Fig. 1. The transfer function matrix from the secondary loudspeakers to the microphones in 
case of coupling, namely $\overline{\mathbf{G}}$, can be represented in terms of the transfer function matrix when coupling does not exist, namely $\mathbf{G}$, as follows:

$$
\overline{\mathbf{G}}=\mathbf{G}_{c}
$$

Proof: By combining equations (50) and (55) the proof is complete".

Lemma 3. For any full-rank $M \times L$ matrix $\mathbf{G}$ and matrix $\mathbf{X}$ of suitable size, the following equality is always true:

$$
\mathbf{G X}\left[\mathbf{X}^{H} \mathbf{G}^{H} \mathbf{G X}\right]^{-1} \mathbf{X}^{H} \mathbf{G}^{H}=\mathbf{G}\left[\mathbf{G}^{H} \mathbf{G}\right]^{-1} \mathbf{G}^{H} \text {. }
$$

Proof: to be able to find the inverse in the left-hand side of (58), $\mathbf{G X}$ must be assumed to be full rank. Multiplying of both sides of equation (58) from right-hand side by $\mathbf{G X}$, and arranging the terms yields:

$$
\mathbf{G X}\left[\mathbf{X}^{H} \mathbf{G}^{H} \mathbf{G X}\right]^{-1}\left[\mathbf{X}^{H} \mathbf{G}^{H} \mathbf{G X}\right]=\mathbf{G}\left[\mathbf{G}^{H} \mathbf{G}\right]^{-1}\left[\mathbf{G}^{H} \mathbf{G}\right] \mathbf{X}
$$

From (59) it is straightforward to verify that both sides are equal to $\mathbf{G X} \bullet$

Theorem 1. The maximum singular value of $\overline{\mathbf{Z}}_{c}(\omega)$ is less than unity almost everywhere, unless the coupled secondary loudspeakers get very close to each other.

Proof: the theorem can be proved by following exactly the same steps as given by reference [16] and hence is not repeated here. In this manner, it can be shown:

$$
\bar{\sigma}\left[\overline{\mathbf{Z}}_{c}(\omega)\right]=\frac{\left|Z_{s}\right|}{\min \left(\sqrt{\left|Z_{s}\right|^{2}+\left|\underline{\sigma}_{c}\right|^{2}}, \sqrt{\lambda_{\text {min }}\left(\operatorname{Re}\left[2 \bar{Z}_{s} \mathbf{Z}_{c}\right]\right)}\right)}
$$

where $\left|Z_{s}\right|$ and $\bar{Z}_{s}$ represent the magnitude and complex conjugate of $Z_{s}$ repectively. Since for a typical loudspeaker and acoustic environment inside the enclosure, the values of $\left|Z_{s}\right|$ at each frequency are much larger than the magnitude of the frequency responses of the enclosure, i.e. elements of $\mathbf{Z}_{c}$, it can be inferred that the value of the expression $\sqrt{\lambda_{\min }\left(\operatorname{Re}\left[2 Z_{s}^{\prime} \mathbf{Z}_{c}\right]\right)}$ is much larger than $\sqrt{\left|Z_{s}\right|^{2}+\left|\underline{\sigma}_{c}\right|^{2}}$, unless the matrix $\mathbf{Z}_{c}$ approaches a singular point, as discussed in Remark 2. From this argument it can be deduced that in normal situations when the loudspeakers are sufficiently far apart, equation (60) is simplified to:

$$
\bar{\sigma}\left[\overline{\mathbf{Z}}_{c}(\omega)\right]=\frac{\left|Z_{s}\right|}{\sqrt{\left|Z_{s}\right|^{2}+\left|\underline{\sigma}_{c}\right|^{2}}}<1
$$

which is less than unity at almost all frequencies when the ASC system is not singular $\bullet$. 
Noting the relationship between the matrices $\mathbf{G}$ and $\overline{\mathbf{G}}$ in (57) obtained in Lemma 2, along with the results of Lemmas 1 and 3, it is possible to evaluate how the active sound field control system will perform to reduce the acoustic potential energy inside the enclosure when coupling exists between loudspeakers. This is formulated with the following theorem:

Theorem 2. Having an array of $L$ loudspeakers and $M$ microphones inside the enclosure as shown in Fig. 1, the following statements are true:

1- In the case that the loudspeaker positions are such that the system is non-singular, the maximum achievable reduction of acoustic noise with coupling among secondary loudspeakers is the same as the amount of reduction when coupling does not exist.

2- In the frequency range between two consecutive zeros of the frequency response function from secondary loudspeakers to error microphones, coupling of loudspeakers will change the amplitude and phase of the optimal voltage applied to the secondary loudspeakers such that $\left\|\mathbf{v}_{s o}\right\|_{2} \leq\left\|\overline{\mathbf{v}}_{\text {so }}\right\|_{2}$.

Proof: The maximum reduction of approximated potential energy inside the enclosure when coupling exists among secondary sources is obtained from equation (52). To prove the first part of Theorem 2, we substitute $\overline{\mathbf{G}}$ from equation (57) into equation (52):

$$
\frac{J_{\mathrm{po}}}{J_{\mathrm{pp}}}=1-\frac{\mathbf{p}^{H} \mathbf{G} \overline{\mathbf{Z}}_{c}\left[\overline{\mathbf{Z}}_{c}^{H} \mathbf{G}^{H} \mathbf{G} \overline{\mathbf{Z}}_{c}\right]^{-1} \overline{\mathbf{Z}}_{c}^{H} \mathbf{G}^{H} \mathbf{p}}{\mathbf{p}^{H} \mathbf{p}}
$$

Applying Lemma 3 to equation (52) yields:

$$
\frac{J_{\mathrm{po}}}{J_{\mathrm{pp}}}=1-\frac{\mathbf{p}^{H} \mathbf{G}\left[\mathbf{G}^{H} \mathbf{G}\right]^{-1} \mathbf{G}^{H} \mathbf{p}}{\mathbf{p}^{H} \mathbf{p}}
$$

This expression is exactly the same as the approximation of acoustic potential energy inside the enclosure when no coupling exists. To prove the second part of Theorem 2, it is necessary to substitute equation (57) into (51). In this case, the optimal voltage applied to the secondary loudspeakers is:

$$
\overline{\mathbf{v}}_{s o}=-\left[\overline{\mathbf{Z}}_{c}^{H} \mathbf{G}^{H} \mathbf{G} \overline{\mathbf{Z}}_{c}\right]^{-1} \overline{\mathbf{Z}}_{c}^{H} \mathbf{G}^{H} \mathbf{p} .
$$

Multiplying both sides of equation (64) by $\mathbf{G} \overline{\mathbf{Z}}_{c}$ yields:

$$
\mathbf{G} \overline{\mathbf{Z}}_{c} \overline{\mathbf{v}}_{s o}=-\mathbf{G} \overline{\mathbf{Z}}_{c}\left[\overline{\mathbf{Z}}_{c}^{H} \mathbf{G}^{H} \mathbf{G} \overline{\mathbf{Z}}_{c}\right]^{-1} \overline{\mathbf{Z}}_{c}^{H} \mathbf{G}^{H} \mathbf{p} .
$$




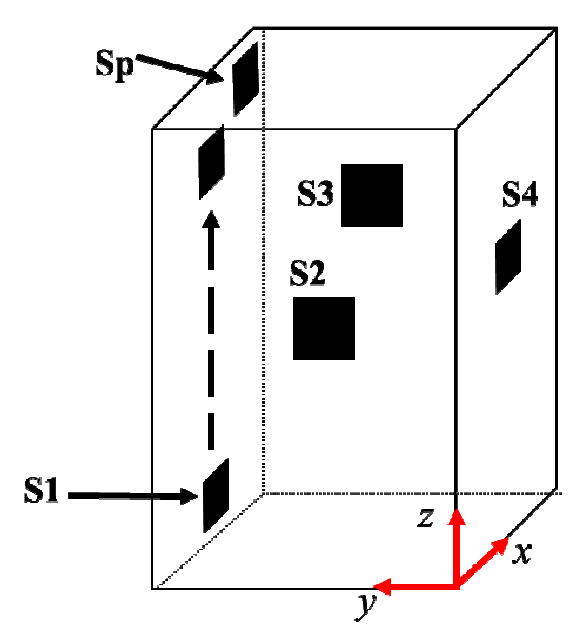

(a)

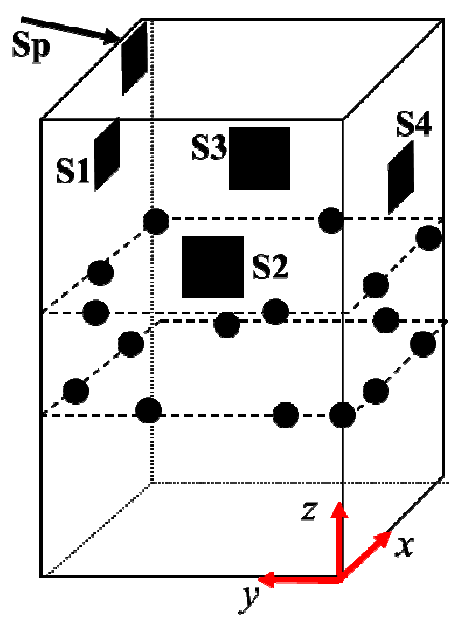

(b)

Figure 2. (a) Schematic of the array of primary and secondary sources in the enclosure, (b) Arrangement of array of secondary loudspeakers and error microphones inside the enclosure.

Using Lemma 3:

$\mathbf{G} \overline{\mathbf{Z}}_{c} \overline{\mathbf{v}}_{s o}=-\mathbf{G}\left[\mathbf{G}^{H} \mathbf{G}\right]^{-1} \mathbf{G}^{H} \mathbf{p}$

Writing the right hand side of equation (66) in terms of $\mathbf{v}_{s o}$ results in,

$\mathbf{G} \overline{\mathbf{Z}}_{c} \overline{\mathbf{v}}_{s o}=\mathbf{G} \mathbf{v}_{s o}$

Under the assumption of having a full rank $\mathbf{G}$ matrix, i.e $\mathbf{G}^{H} \mathbf{G}$ is invertible, it can be readily inferred that,

$\mathbf{v}_{s o}=\overline{\mathbf{Z}}_{c} \overline{\mathbf{v}}_{s o}$

According to (68) it can be seen that coupling will change the amplitude and phase of secondary loudspeakers due to the effect of the coupling matrix $\overline{\mathbf{Z}}_{c}$. As the maximum singular value of $\overline{\mathbf{Z}}_{c}$ is less than unity in most of the in-bandwidth frequencies of the system (according to Theorem 1), if the coupled loudspeakers are far enough apart, it is deduced that the $\infty$-norm of $\overline{\mathbf{Z}}_{c}$ between two consecutive zeros of the frequency response function of the system is less than unity and hence $\left\|\mathbf{v}_{s o}\right\|_{2} \leq\left\|\overline{\mathbf{v}}_{s o}\right\|_{2} \cdot$ 


\section{Performance Analysis in Extreme Cases}

For a better insight on the performance of the system in extreme cases a further analytical study is conducted in this section. By the extreme case we mean the conditions under which the applied voltages to the loudspeakers in (51) go to infinity or will be beyond an acceptable range. We distinguish two cases when the transfer matrix $\overline{\mathbf{G}}$ loses rank at a specific frequency and the case when $\overline{\mathbf{G}}$ loses rank due to observability or controllability of a specific mode.

\subsection{High Control Effort at Specific Frequencies}

High control effort in equation (51) might occur in two cases: 1- Right half plane zeros of the transfer matrix $\overline{\mathbf{G}}$ which might appear as right half plane poles in (51) due to the matrix inversion. 2- Increase in the control action $\mathbf{v}_{\mathrm{eo}}$ when the matrix $\overline{\mathbf{G}}$ loses rank or at frequencies where the matrix $\overline{\mathbf{G}}$ has a small magnitude. To address the first point we use an inner-outer factorization technique. The basic idea behind the inner-outer (or outer-inner factorization) is to factorize dynamic systems in a part which is stably (causally) invertible (the outer factor) and a remaining part (the inner factor) which only yields a phase shift (e.g. due to delays) and thus does not affect the energy of the signals.

Lemma 4 (Outer-Inner factorization [26]). Let $\overline{\mathbf{G}} \in R H_{\infty}^{M \times L}$ then $\overline{\mathbf{G}}$ has an inner-outer factorization

$$
\overline{\mathbf{G}}=\overline{\mathbf{G}}_{i} \overline{\mathbf{G}}_{o}
$$

with $\overline{\mathbf{G}}_{i} \in R H_{\infty}^{M \times L}$ is an isometry $\left(\overline{\mathbf{G}}_{i}^{\mathrm{H}} \overline{\mathbf{G}}_{i}=I_{M}\right)$ and the outer factor $\overline{\mathbf{G}}_{o} \in R H_{\infty}^{M \times L}$ has a stable right inverse, with $m \leq \min (M, L)$. If the transfer matrix $\overline{\mathbf{G}}$ does not lose rank on the imaginary axis then $\overline{\mathbf{G}}_{o}$ has an asymptotically stable right inverse. By substituting $\overline{\mathbf{G}}$ from (69) to equation (51) and using the isometry property of $\overline{\mathbf{G}}_{i}$ it can be written as

$$
\overline{\mathbf{v}}_{\mathrm{eo}}=-\left[\overline{\mathbf{G}}_{o}{ }^{H} \overline{\mathbf{G}}_{o}\right]^{-1} \overline{\mathbf{G}}^{H} \mathbf{p}_{\mathrm{p}}
$$

Since $\overline{\mathbf{G}}_{o}$ has a stable right inverse it is said to be minimum-phase and as long as the transfer matrix $\overline{\mathbf{G}}$ does not lose rank at a specific frequency it will also have a stable left inverse and hence $\overline{\mathbf{v}}_{\mathrm{eo}}$ in (70) is finite. To address the problem of singularity of the transfer matrix $\overline{\mathbf{G}}$ at specific frequencies another performance index needs to be introduced. The proposed performance index is in the following form 
$J_{\mathrm{P}}=\frac{V}{4 \rho_{0} c_{0}^{2} M} \mathbf{p}^{\mathrm{H}} \mathbf{p}+\mathbf{v}_{e}^{\mathrm{H}} \tilde{\mathbf{v}}_{e}$

where $\tilde{\mathbf{v}}_{e}$ is the filtered control signal and is given by

$\tilde{\mathbf{v}}_{e}=\mathbf{W} \mathbf{v}_{e}$

Here $\mathbf{W}$ is an $L \times L$ user defined filter and gives the freedom to restrict the control signal at specific frequencies. Minimization of the new performance index (71) yields the optimal excitation voltage of the secondary loudspeakers in case of coupling among secondary sources

$\overline{\mathbf{v}}_{\mathrm{so}}=-\left[\overline{\mathbf{G}}^{H} \overline{\mathbf{G}}+\mathbf{W}^{H} \mathbf{W}\right]^{-1} \overline{\mathbf{G}}^{H} \mathbf{p}_{\mathrm{p}}$

$\frac{J_{\mathrm{po}}}{J_{\mathrm{pp}}}=1-\frac{\mathbf{p}_{\mathrm{p}}{ }^{H} \overline{\mathbf{G}}\left[\overline{\mathbf{G}}^{H} \overline{\mathbf{G}}+\mathbf{W}^{H} \mathbf{W}\right]^{-1} \overline{\mathbf{G}}^{H} \mathbf{p}_{\mathrm{p}}}{\mathbf{p}_{\mathrm{p}}{ }^{H} \mathbf{p}_{\mathrm{p}}}$

As can be seen from (72) at the frequencies where the transfer matrix $\overline{\mathbf{G}}$ loses rank it is possible to increase the magnitude of the weight matrix $\mathbf{W}$ to solve the singularity in matrix inversion and thus avoid high control efforts. This is of course at the expense of reducing the performance of the system in (73). A simpler version of this technique when the filter $\mathbf{W}$ is replaced by a scalar is investigated by the first author in [15] for optimal placement of loudspeakers and microphones considering coupling of secondary sources.

\subsection{Controllability and Observability of Modes}

Modelling and analysis carried out in section 3 facilitates studying of the optimal placement of loudspeakers and microphones for the active sound field control problem. This problem is addressed in a number of investigations by the first author [27, 28], and in [29] by considering the coupling of the secondary loudspeakers. In all of these papers the optimization problem is solved by choosing a quadratic performance index and, after finding the control law, position for loudspeakers and microphones is optimized to achieve the maximum potential energy reduction. In this sense, the optimization is carried out in closed-loop. An alternative view to the problem of loudspeaker and microphone placement is to use the notion of modal controllability and observability. It is well-known that the control effort of the designed control system is directly related to the controllability and observability of the in bandwidth modes of the system under control [31]. The controllability and observability of the transfer matrix $\overline{\mathbf{G}}$ can be determined by checking the rank of controllability and observability matrices for a specific realization of $\overline{\mathbf{G}}$. However, a more quantitative measure to determine how hard it is to control or observe a specific mode is to use the concept of Gramians in control theory. Before being able to calculate a 
measure of controllability and observability of the acoustic modes, a state space realization of the transfer matrix $\overline{\mathbf{G}}$ should be available.

By combining equations (50) and (55), it can be seen that $\overline{\mathbf{G}}$ is written as a series connection of two transfer matrices, $\mathbf{G}$ and $\overline{\mathbf{Z}}_{c}$

$\overline{\mathbf{G}}=\boldsymbol{\Psi}^{\mathrm{T}} \mathbf{B} \overline{\mathbf{Z}}_{c}=\mathbf{G} \overline{\mathbf{Z}}_{c}$

By element-wise multiplication of the matrices $\boldsymbol{\Psi}$ and $\mathbf{B}$, the transfer matrix $\mathbf{G}$ in (74) can be written as

$\mathbf{G}(\omega)=\sum_{n=1}^{N} A_{n}(\omega) \boldsymbol{\Psi}_{n} \boldsymbol{\alpha}_{n}^{\mathrm{T}}$

Where $\boldsymbol{\psi}_{n}$ and $\boldsymbol{\alpha}_{n}$ are defined as $M \times 1$ and $L \times 1$ vectors

$\boldsymbol{\psi}_{n}=\left[\psi_{n}\left(x, y_{1}, z\right), \psi_{n}\left(x, y_{2}, z\right), \ldots \ldots, \psi_{n}\left(x, y_{M}, z\right)\right]^{\mathrm{T}}$

$\boldsymbol{\alpha}_{n}=\left[\alpha_{n 1}, \alpha_{n 2}, \ldots \ldots, \alpha_{n L}\right]^{\mathrm{T}}$

$\alpha_{n i}=\int_{\mathrm{SP}_{\mathrm{i}}} \psi_{n}\left(x, y_{i}, z\right) \mathrm{d} x \mathrm{~d} z$

and $A_{n}(\omega)$ is defined in (7). According to (75) the state space realization of $\mathbf{G}$ can be written as $\mathbf{A}=\left[\begin{array}{cc}\mathbf{0}_{N \times N} & \mathbf{I}_{N \times N} \\ \mathbf{A}_{1 N \times N} & \mathbf{A}_{2 N \times N}\end{array}\right]$

$\mathbf{A}_{1}=-\operatorname{diag}\left(\begin{array}{llll}\omega_{1}^{2} & \omega_{2}^{2} & \ldots & \omega_{N}^{2}\end{array}\right)$

$\mathbf{A}_{2}=-2 \operatorname{diag}\left(\xi_{1} \omega_{1} \quad \xi_{2} \omega_{2} \quad \ldots \quad \xi_{N} \omega_{N}\right)$

$\mathbf{B}=\left[\begin{array}{c}\mathbf{0}_{N \times L} \\ \boldsymbol{\alpha}_{1}^{\mathrm{T}} \\ \vdots \\ \boldsymbol{\alpha}_{N}^{\mathrm{T}}\end{array}\right], \mathbf{C}=\left[\boldsymbol{0}_{M \times N}, \boldsymbol{\psi}_{1}, \boldsymbol{\psi}_{2}, \ldots ., \boldsymbol{\psi}_{N}\right]$

Before being able to find the state space realization for the transfer matrix $\overline{\mathbf{Z}}_{c}$, equation (54) should be rewritten in a suitable form. This is achieved by combining the scalar factor $Z_{s}(\omega)$ with the transfer matrix $\mathbf{Z}_{c}(\omega)$, and hence it can be rewritten as

$\overline{\mathbf{Z}}_{c}(\omega)=\left(\mathbf{I}+Z_{s}^{-1}(\omega) \mathbf{Z}_{c}(\omega)\right)^{-1}$ 
It is possible to find the state space realization of the transfer matrix $\mathbf{Z}_{c}$ in (80) by putting all elements defined in (25) in the following matrix form

$\mathbf{Z}_{c}(\omega)=\sum_{n=1}^{N} A_{n}(\omega) \boldsymbol{\alpha}_{n} \boldsymbol{\alpha}_{n}^{\mathrm{T}}$

where $\boldsymbol{\alpha}_{n}$ is defined in (77) and (78). The state space realization for $\mathbf{Z}_{c}$ in (81) is similar to the one achieved for the transfer matrix $\mathbf{G}$ in (75) except the matrix $\mathbf{C}$ is changed. The state space realization for the transfer matrix $\mathbf{Z}_{c}$ can be written as

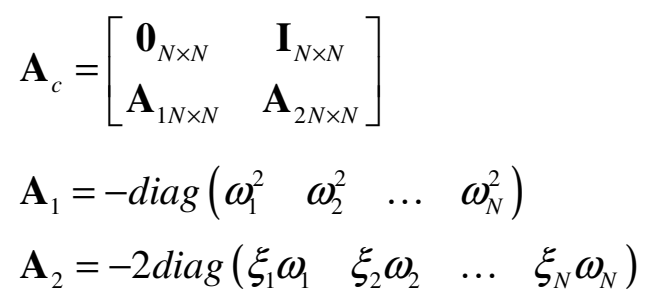

$\mathbf{B}_{c}=\left[\begin{array}{c}\mathbf{0}_{N \times L} \\ \boldsymbol{\alpha}_{1}^{\mathrm{T}} \\ \vdots \\ \boldsymbol{\alpha}_{N}^{\mathrm{T}}\end{array}\right], \mathbf{C}_{c}=\left[\mathbf{0}_{L \times N}, \boldsymbol{\alpha}_{1}, \boldsymbol{\alpha}_{2}, \ldots ., \boldsymbol{\alpha}_{N}\right]$

By having the state space realization of $\mathbf{Z}_{c}$ in (82), it would be enough to find a realization for the third order strictly proper transfer function $Z_{s}^{-1}(\omega)$. Therefore, a state space realization of the transfer matrix $Z_{s}^{-1}(\omega) I_{L}$ can be written as

$$
\mathbf{A}_{\mathrm{spk}}=A_{s} \otimes I_{L}, \mathbf{B}_{\mathrm{spk}}=B_{s} \otimes I_{L}, \mathbf{C}_{\mathrm{spk}}=C_{s} \otimes I_{L} \text {, and } \mathbf{D}_{\mathrm{spk}}=0_{L \times L}
$$

where $\left(A_{s}, B_{s}, C_{s}\right)$ is a minimal realization of $Z_{s}^{-1}(\omega)$ and $\otimes$ is the Kronecker product. The matrices $A_{s}, B_{s}, C_{s}$ are $3 \times 3,3 \times 1$ and $1 \times 3$ respectively. In this way, the state space realization of the transfer matrix achieved from series connection of $\mathbf{Z}_{c}$ followed by $Z_{s}^{-1}(\omega) I_{L}$ can be written as

$$
\begin{aligned}
& \mathbf{A}_{\text {series }}=\left[\begin{array}{cc}
\mathbf{A}_{c} & 0_{2 N \times 3 L} \\
\mathbf{B}_{\text {spk }} \mathbf{C}_{c} & \mathbf{A}_{\text {spk }}
\end{array}\right] \\
& \mathbf{B}_{\text {series }}=\left[\begin{array}{c}
\mathbf{B}_{c} \\
0_{3 L \times L}
\end{array}\right], \mathbf{C}_{\text {series }}=\left[\begin{array}{ll}
0_{L \times 2 N} & \mathbf{C}_{s p k}
\end{array}\right], \mathbf{D}_{\text {series }}=0_{L \times L}
\end{aligned}
$$

Therefore, the state space realization of the feedback system represented by $\overline{\mathbf{Z}}_{c}$ can be found as 


$$
\mathbf{A}_{\bar{Z}_{c}}=\left[\begin{array}{cc}
\mathbf{A}_{c} & \mathbf{B}_{c} \mathbf{C}_{\mathrm{spk}} \\
\mathbf{B}_{\mathrm{spk}} \mathbf{C}_{c} & \mathbf{A}_{\mathrm{spk}}
\end{array}\right], \mathbf{B}_{\bar{Z}_{c}}=-\left[\begin{array}{c}
\mathbf{B}_{c} \\
0
\end{array}\right], \mathbf{C}_{\bar{Z}_{c}}=-\left[\begin{array}{ll}
0 & \mathbf{C}_{s \mathrm{pk}}
\end{array}\right], \quad \mathbf{D}_{\bar{Z}_{c}}=0_{L \times L}
$$

In steady state conditions, the controllability Gramian matrix $\mathbf{W}_{c}$, and the observability Gramian matrix $\mathbf{W}_{\mathrm{o}}$ can be determined by solving the following Lyapunov equations [32].

$$
\begin{aligned}
& \mathbf{A}_{\bar{Z}_{c}} \mathbf{W}_{\mathrm{c}}+\mathbf{W}_{\mathrm{c}} \mathbf{A}_{\bar{Z}_{c}}^{\mathrm{T}}+\mathbf{B}_{\bar{Z}_{c}} \mathbf{B}_{\bar{Z}_{c}}^{\mathrm{T}}=0 \\
& \mathbf{A}_{\bar{Z}_{c}}^{\mathrm{T}} \mathbf{W}_{\mathrm{o}}+\mathbf{W}_{\mathrm{o}} \mathbf{A}_{\bar{Z}_{c}}+\mathbf{C}_{\bar{Z}_{c}}^{\mathrm{T}} \mathbf{C}_{\bar{Z}_{c}}=0
\end{aligned}
$$

where the state space matrices $\left(\mathbf{A}_{\bar{Z}_{c}}, \mathbf{B}_{\bar{Z}_{c}}, \mathbf{C}_{\bar{Z}_{c}}\right)$ represent a realization of the transfer matrix $\overline{\mathbf{G}}$ derived in (85). Although Gramians depend on the state-space realization, the eigenvalues of the product $\mathbf{W}_{\mathrm{c}} \mathbf{W}_{\mathrm{o}}, \lambda_{i}(i=1,2, \ldots N)$ are invariant under the coordinate transformations and provide valuable information regarding the system controllability and observability. In order to show how the position of loudspeakers and microphones affect the degree of controllability or observability of different modes, the system Hankel Singular Values (HSV) are defined as

$$
\sigma_{i}^{\mathrm{H}}=\sqrt{\lambda_{i}}
$$

where $\lambda_{i} \mathrm{~s}$ are ordered to obtain $\sigma_{1}^{\mathrm{H}} \geq \sigma_{2}^{\mathrm{H}} \geq \cdots \geq \sigma_{N}^{\mathrm{H}}$. The number of non-zero HSV corresponds to the number of controllable and observable modes and their magnitude show the degree of controllability and observability of each mode. An identical approach for optimization of PZT sensors and actuators on a flexible plate is reported in [30].

\section{SIMULATION RESULTS}

To verify the theoretical results presented in the previous sections, a simulation study is performed here. All simulations are carried out in MALTAB and are based on the analytical derivations presented in the previous section. The simulations are divided into two parts: in the first part it is assumed that only loudspeakers are included in the enclosure, and the modal information of the enclosure is known.

For the second part, both loudspeakers and microphones are considered to simulate a practical coupled multichannel ASC system. In both cases the effect of coupling on both resonance and non-resonance frequencies of the enclosure is investigated. Figure 2 illustrates the arrangement of the primary and secondary loudspeakers as well as microphones used in the simulation of the ASC system. To facilitate a better understanding of the effect of coupling inside the enclosure the numerical results in all cases are compared with and without coupled sources. 


\subsection{Array of loudspeakers in enclosure}

To show how the position of secondary sources may affect the performance of the ASC system, the configuration of loudspeakers shown in Fig. 2 (a) is used for simulation. An array of three loudspeakers is placed on three different walls of the enclosure at the height $1.8 \mathrm{~m}$ and the source $\mathrm{S} 1$ is moving in the $z$-direction towards the primary source $\mathrm{Sp}$, half-way through the same plane. The reduction of potential energy inside the enclosure for both coupled and uncoupled secondary sources (equation (47)) when the primary source excites the enclosure at the resonance frequencies $85 \mathrm{~Hz}$ and $170 \mathrm{~Hz}$ and non-resonance frequencies $120 \mathrm{~Hz}$ and $300 \mathrm{~Hz}$ are shown in Fig. 3. As can be seen in this figure, when S1 moves towards Sp, reduction of potential energy for

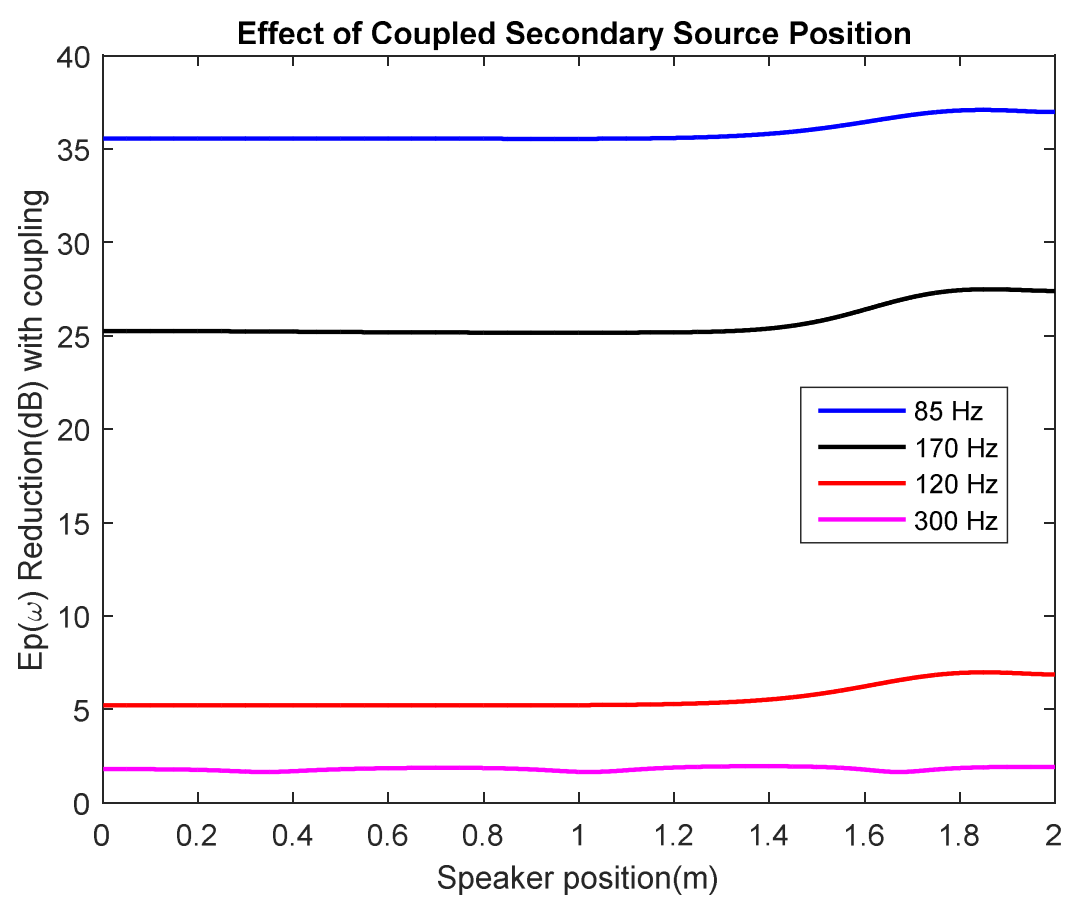

Figure 3. The performance of ASC system when S1 moves towards Sp at different resonant and nonresonant frequencies in Fig. 2(a). 

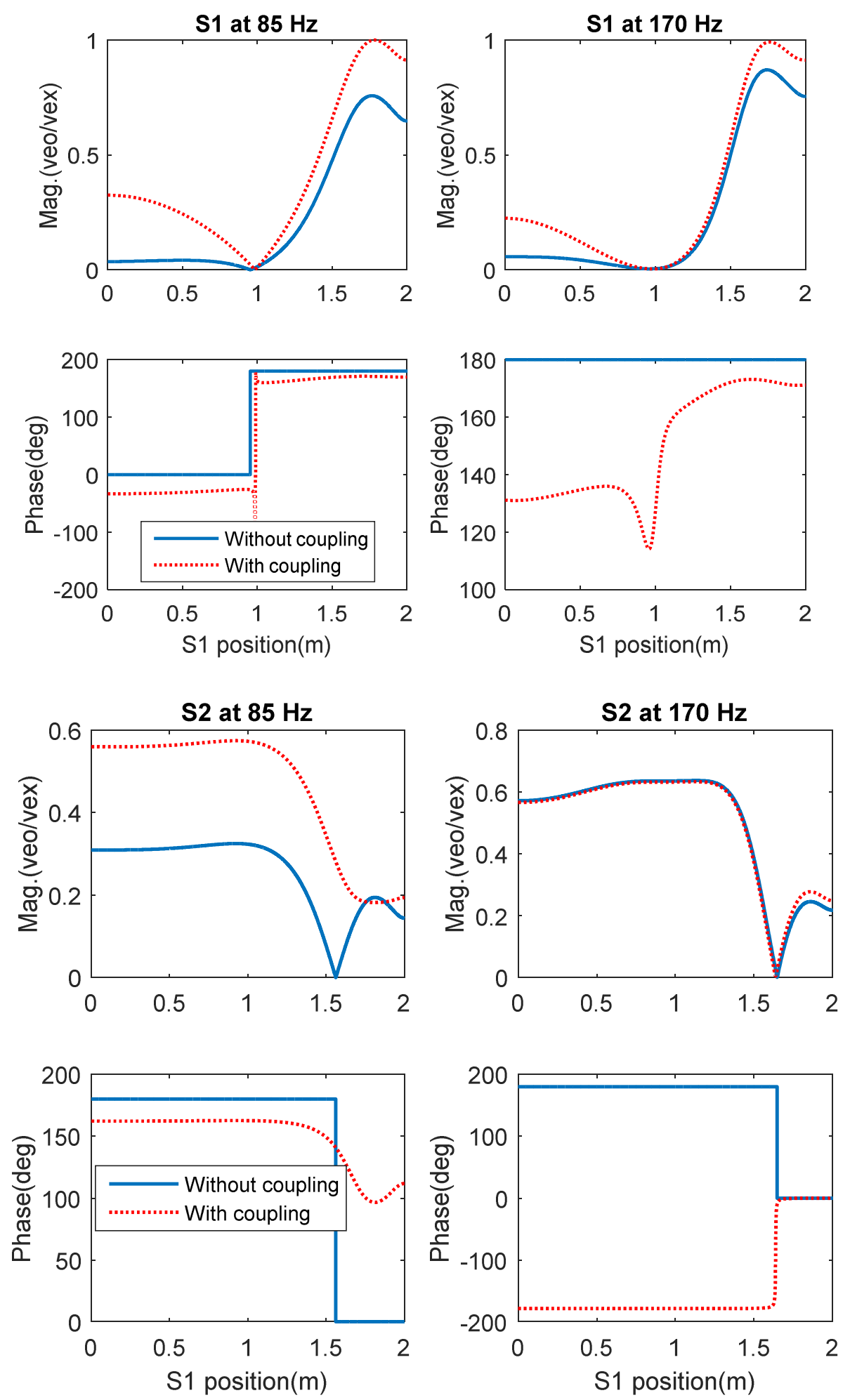

Figure 4. The magnitude and phase of optimal excitation signal of S1 (top) and S2 (bottom) in terms of S1 speaker position at two resonant frequencies of the enclosure, i.e. $85 \mathrm{~Hz}$ and $170 \mathrm{~Hz}$. 

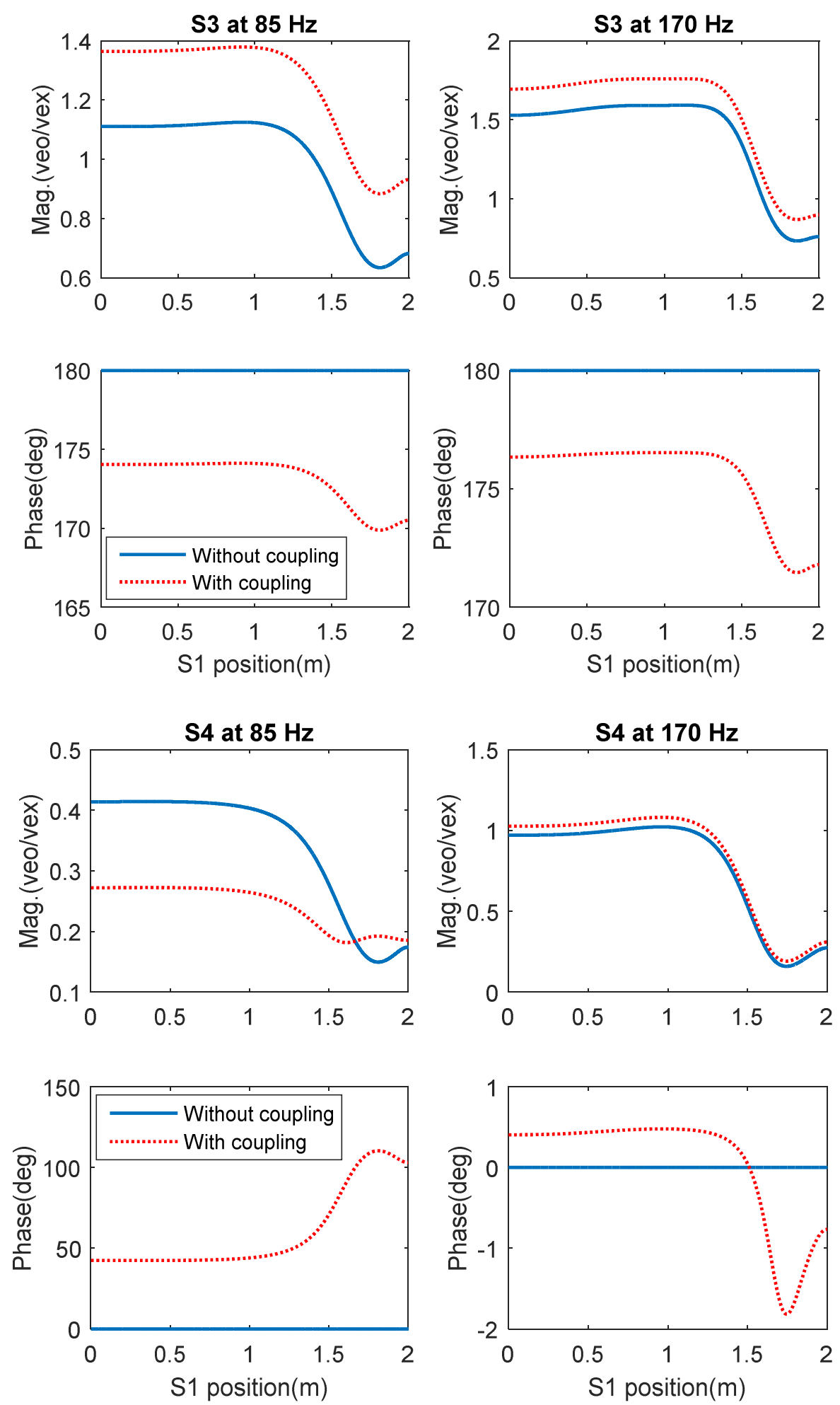

Figure 5. The magnitude and phase of optimal excitation signal of S3 (top) and S4 (bottom) in terms of S1 speaker position at two resonant frequencies of the enclosure, i.e. $85 \mathrm{~Hz}$ and $170 \mathrm{~Hz}$. 
both coupled and uncoupled sources is the same for all frequencies. This is in accordance with the first part of Theorem 2 formulated in section 3.3. Figures 4 and 5 depict the optimal magnitude and phase of four secondary loudspeakers as a function of the position of loudspeaker S1 for both coupled and uncoupled case for two resonance frequencies.

Analysis in [14] revealed that the response of the enclosure at $85 \mathrm{~Hz}$ is dominated by one axial mode shape while at $170 \mathrm{~Hz}$ this is obtained from a combination of three axial modes. In the nonresonant modes $120 \mathrm{~Hz}$ and $300 \mathrm{~Hz}$ this requires cancellation of an even higher number of modes by a greater number of loudspeakers to achieve the same amount of reduction. This explains the reason for the highest reduction at $85 \mathrm{~Hz}$ and the lowest reduction at $300 \mathrm{~Hz}$ in Fig. 3. The magnitude and phase of all four secondary sources (equation (46)) as a function of the position of S1 for both coupled and uncoupled cases are shown in Figs. 4 to 7 . The magnitudes are normalized by the supplied excitation voltage of the primary source to demonstrate their relative values. As can be seen from Figs. 4 and 5, in both resonant frequencies, when S1 is far away from the primary loudspeaker $\mathrm{Sp}$, the optimal relative magnitude of the voltage applied to this loudspeaker is very small compared to the other three loudspeakers. However, when S1 is getting close to $\mathrm{Sp}$ its magnitude relative to Sp rises gradually and this corresponds to the reduction of magnitude of three other sources to compromise the effect of S1. A closer look at the phase of the voltages of loudspeakers for uncoupled case shows that for the resonance frequency $85 \mathrm{~Hz}, \mathrm{~S} 2$ and $\mathrm{S} 3$ are predominantly canceling the mode shape at this frequency and the role of S4 is to absorb the extra energy produced by other loudspeakers. This is also true in the case of couple, except that the magnitude of the applied voltages is greater as predicted by the second part Theorem 2 . At the resonant frequency $170 \mathrm{~Hz}$ due to the existence of degenerate mode shapes, all three loudspeakers S2, S3, and S4 are tuned properly to cancel the effect of these three mode shapes. This is seen from Figs. 4 and 5 by noting that the amplitude of the voltages applied to all loudspeakers is almost the same in both coupled and uncoupled cases. Nonetheless, when S1 gets close to $\mathrm{Sp}$ the rise in voltage amplitude of $\mathrm{S} 1$ is equalized by reduction of the amplitude of voltages of other loudspeakers, and hence all four loudspeakers contribute in cancelling the mode shapes of this frequency. The results for the non-resonant frequencies $120 \mathrm{~Hz}$ and $300 \mathrm{~Hz}$ are shown in Figs. 6 and 7. Analysis shown in [14] reveals that the response of the enclosure at $120 \mathrm{~Hz}$ is mostly dominated by the modes of the resonant frequencies at $170 \mathrm{~Hz}$ with some contributions from $85 \mathrm{~Hz}$. Therefore, it is worthy to note that the change in amplitude and phase of sources S1 to S4 as a function of the position of source S1 is similar to what is shown in Figs. 4 and 5 for the resonant frequency $170 \mathrm{~Hz}$. 

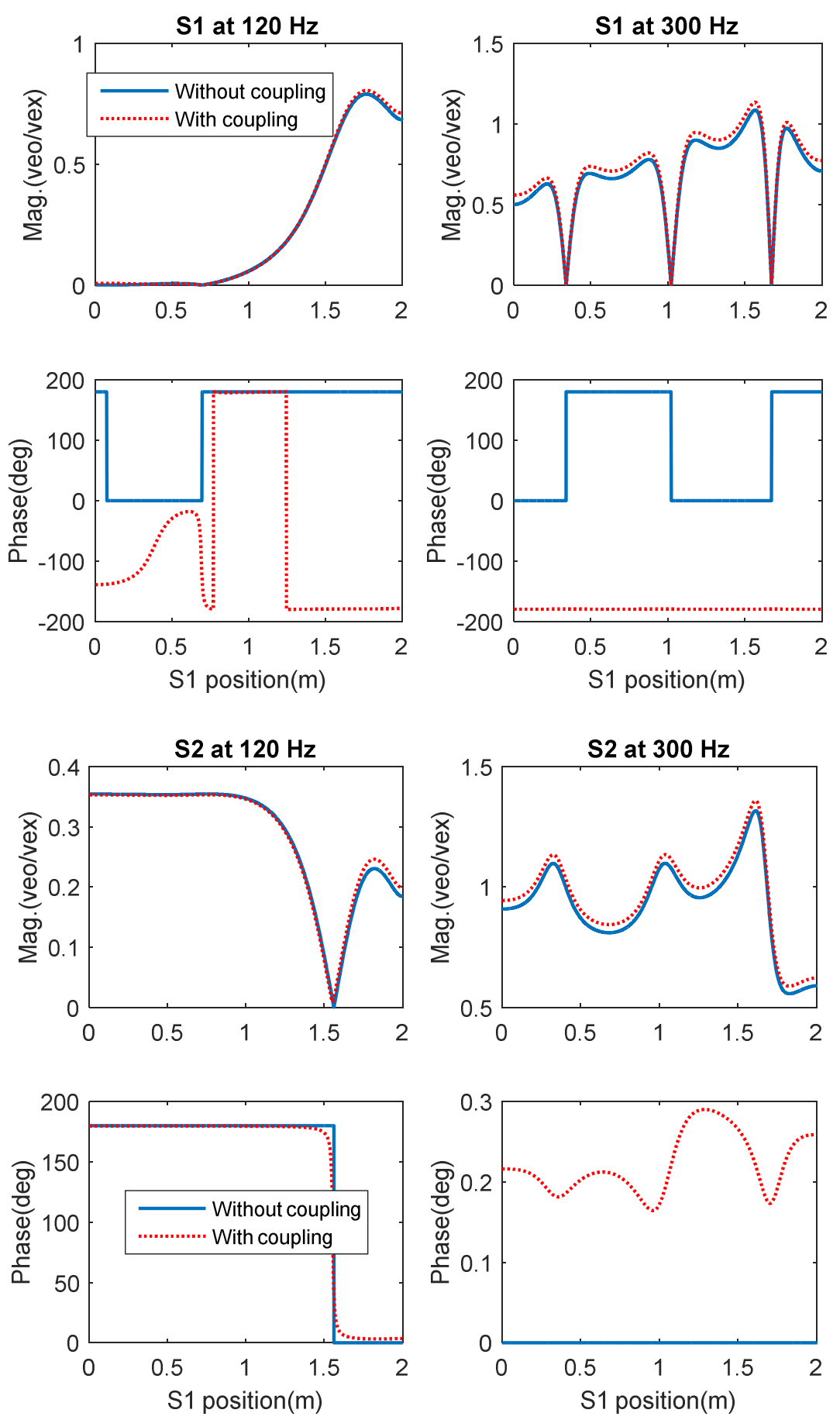

Figure 6. The magnitude and phase of optimal excitation signal of S1 (top) and S2 (bottom) in terms of S1 speaker position at two non-resonant frequencies of the enclosure, i.e. $120 \mathrm{~Hz}$ and $300 \mathrm{~Hz}$. 

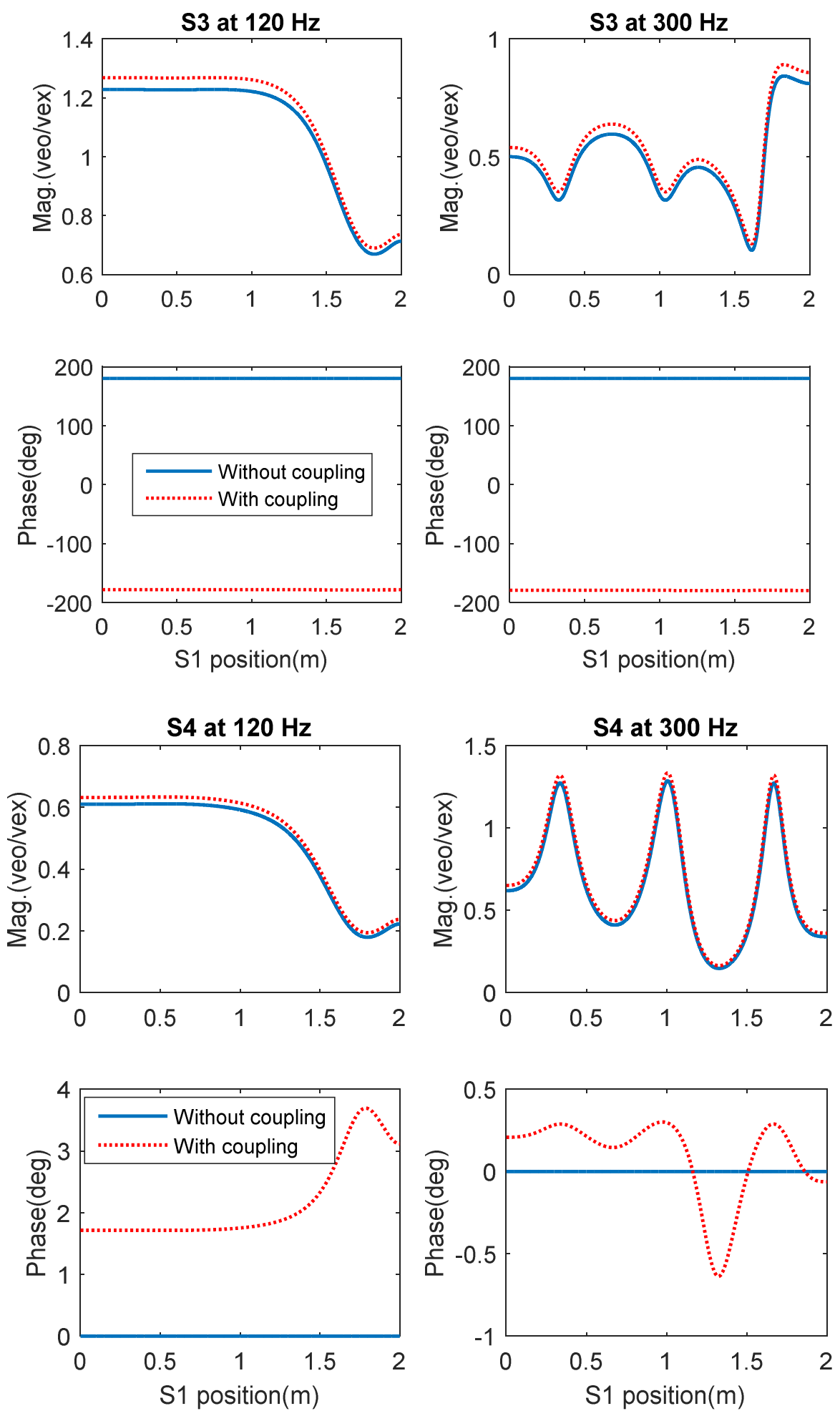

Figure 7. The magnitude and phase of optimal excitation signal of S3 (top) and S4 (bottom) in terms of S1 speaker position at two non-resonant frequencies of the enclosure, i.e. $120 \mathrm{~Hz}$ and $300 \mathrm{~Hz}$. 

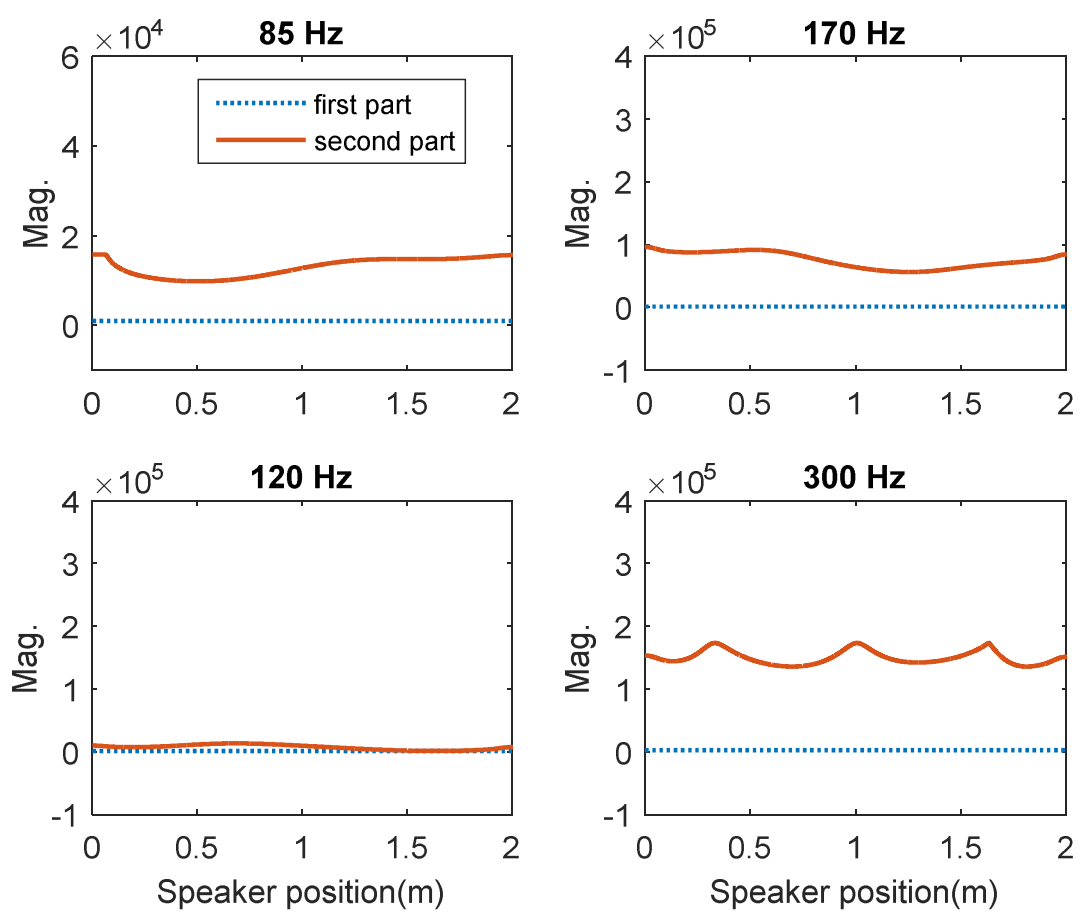

Figure 8. Magnitudes of the first and second parts of (58) at $85 \mathrm{~Hz}, 170 \mathrm{~Hz}, 120 \mathrm{~Hz}$, and $300 \mathrm{~Hz}$.
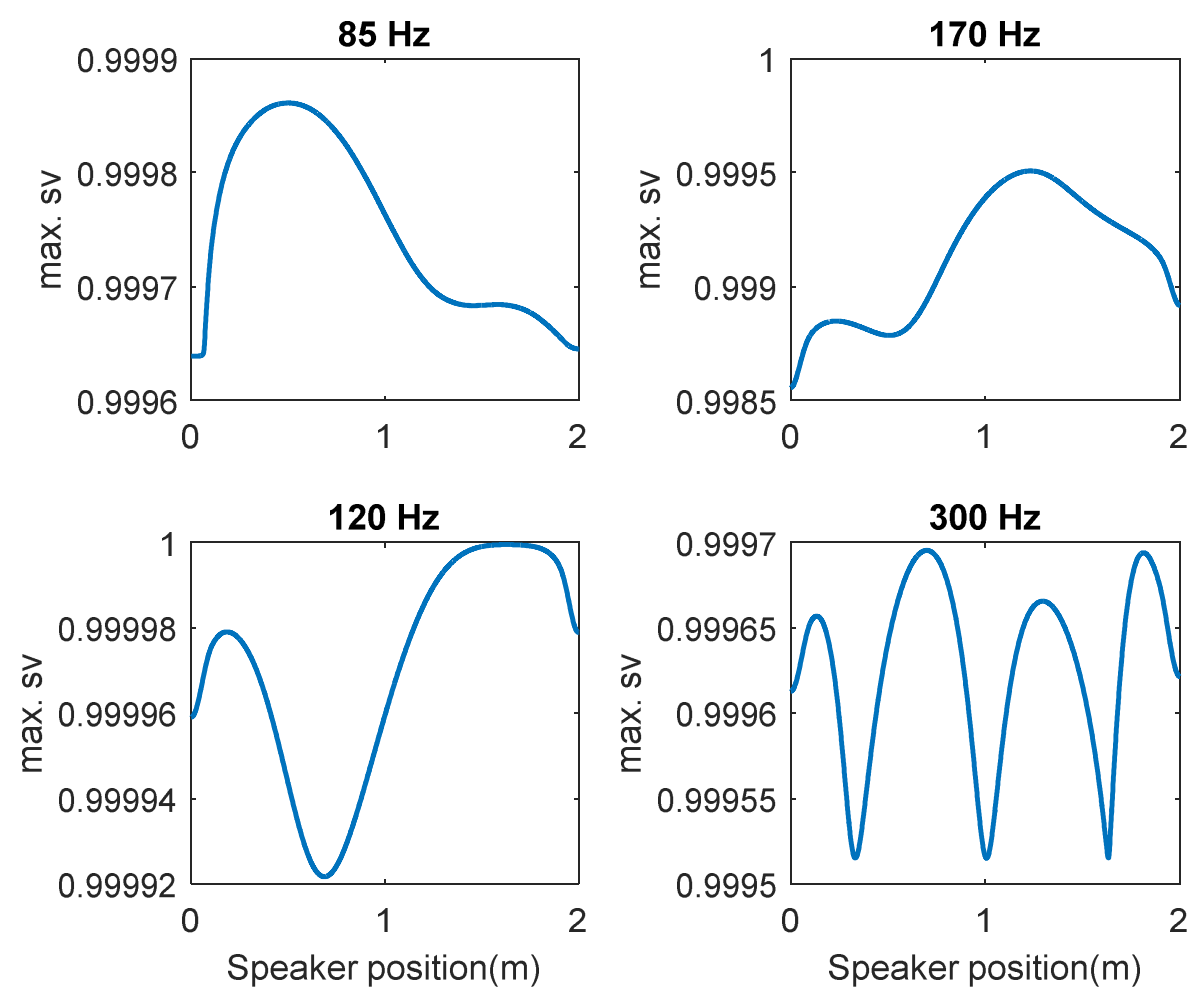

Figure 9. Maximum singular value of the coupling matrix $\overline{\mathbf{Z}}_{c}$ at $85 \mathrm{~Hz}, 170 \mathrm{~Hz}, 120 \mathrm{~Hz}, 300 \mathrm{~Hz}$. 
The response of the enclosure at non-resonant frequency $300 \mathrm{~Hz}$ is dominated by $295 \mathrm{~Hz}$ with some contributions from the resonance frequencies at $255 \mathrm{~Hz}$. Therefore in both coupled and uncoupled cases S2 and S3 are cancelling the effect of each other while S1 and S4 are tuned to cancel the effect of the resonant modes. This is carried out in the coupled case by spending more energy and having a continuous change in the phase of the voltages applied to the secondary loudspeakers compared to the uncoupled loudspeakers. Simulation results show that when two secondary loudspeakers are very close to each other since the matrices $\mathbf{B}$ and $\overline{\mathbf{B}}$ are near to lose rank the optimal voltage will be much larger than the excitation signal applied to the primary loudspeaker.

To understand how coupling affects the performance of the system, the magnitude of the first and second parts of the denominator of (58) for both resonant and non-resonant frequencies are plotted as a function of the position of $\mathrm{S} 1$ in Fig. 8. As can be seen from this figure, for both resonant and non-resonant frequencies the magnitude of the second part is far greater than the first part, except at $120 \mathrm{~Hz}$. This is due to the fact that this frequency is very close to the zeros of the transfer matrix of the system $\mathbf{Z}_{c}$ and hence the second term tends to zero. Closer examination of this plot reveals that due to this fact the magnitude of the second part becomes very close to the first part at some positions. Hence, computation of the maximum singular value of $\overline{\mathbf{Z}}_{c}$ as illustrated in Fig. 9, shows that it is always less than one for all resonant and non-resonant frequencies, although this value is almost equal to one for $120 \mathrm{~Hz}$. This numerical simulation demonstrates the validity of the Theorem 1 about the magnitude of the coupling matrix.

\subsection{Including microphones}

To realize a practical ASC system microphones are added to the enclosure and the performance of a MIMO ASC system is simulated. The configuration of primary, secondary loudspeakers, and error microphones is shown in Fig. 2 (b). As can be seen in this figure, an array of four secondary loudspeakers, one on each wall of the enclosure is placed at the height of $1.8 \mathrm{~m}$ of the enclosure. To measure the potential energy distributed inside the enclosure an array of 16 microphones are placed around the enclosure at two different levels. The selected heights correspond to the peaks of different modes contributing to the acoustic response of the enclosure. In this case 64 transfer functions in total between each pair of loudspeakers and microphones are calculated. The corresponding coordinates of the loudspeakers and microphones are listed in Table 2 and 3. The optimal magnitude and phase of the secondary loudspeakers S1 to S4 and the 


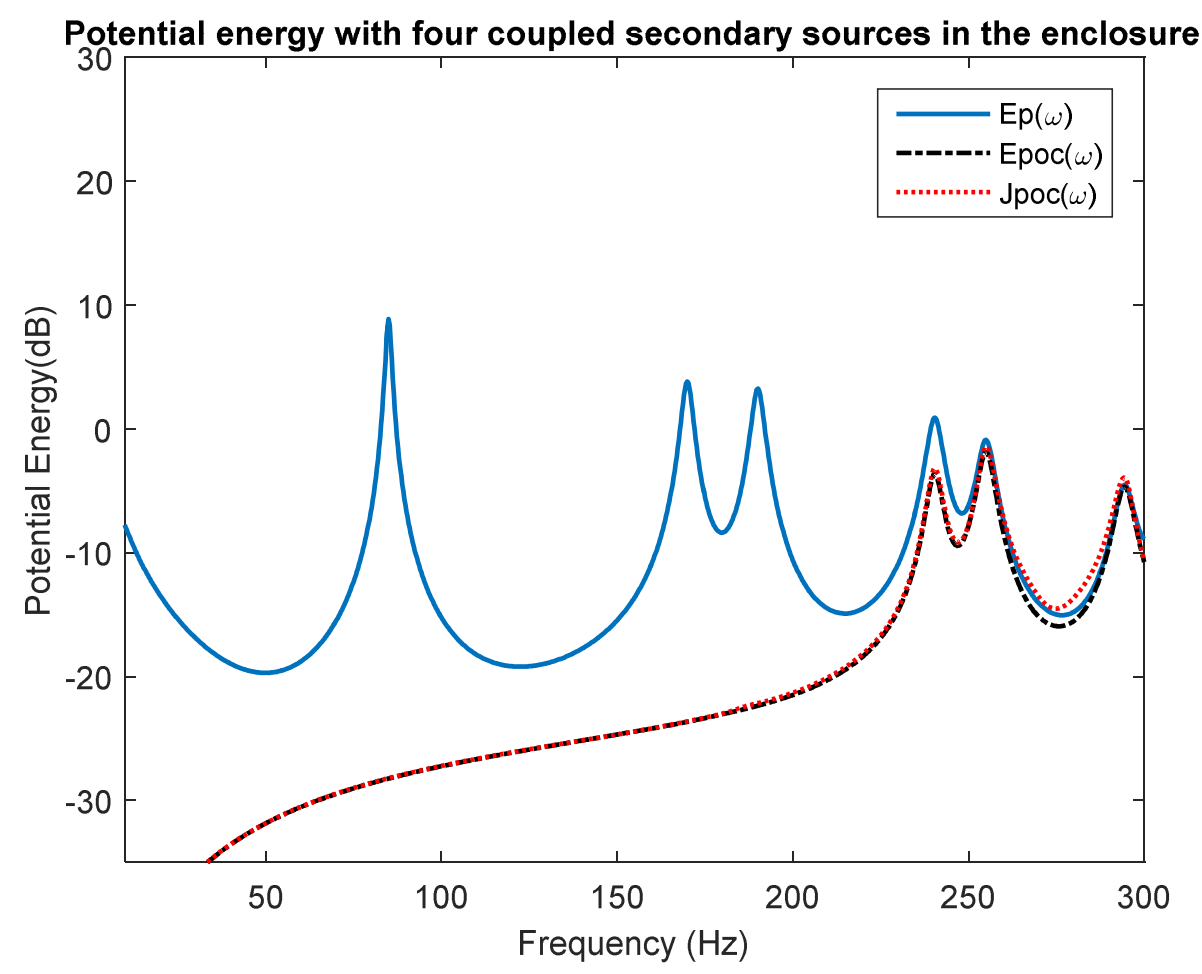

Figure 10. potential energy inside enclosure (blue, solid trace), potential energy inside the enclosure when $E_{p}$ is minimized by knowing the modal information of the enclosure without coupling (black, dashed trace), and potential energy inside enclosure when sixteen microphones are used to minimize $J_{p}$ considering coupling (red, dashed-dot line).

Table 2 Coordinates of array of error microphones inside enclosure.

\begin{tabular}{|c|c|c|c|}
\hline Microphones & Position & Microphones & Position \\
\hline Error microphone 1 & {$[0.075,0.075,1.5]$} & Error microphone 9 & {$[0.075,0.875,1.5]$} \\
\hline Error microphones 2 & {$[0.275,0.075,1]$} & Error microphones 10 & {$[0.875,0.275,1]$} \\
\hline Error microphones 3 & {$[0.475,0.075,1.5]$} & Error microphones 11 & {$[0.875,0.475,1.5]$} \\
\hline Error microphones 4 & {$[0.675,0.075,1]$} & Error microphones 12 & {$[0.875,0.675,1]$} \\
\hline Error microphones 5 & {$[0.875,0.075,1.5]$} & Error microphones 13 & {$[0.875,0.875,1.5]$} \\
\hline Error microphones 6 & {$[0.075,0.275,1]$} & Error microphones 14 & {$[0.275,0.875,1]$} \\
\hline Error microphones 7 & {$[0.075,0.475,1.5]$} & Error microphones 15 & {$[0.475,0.875,1.5]$} \\
\hline Error microphones 8 & {$[0.075,0.675,1]$} & Error microphones 16 & {$[0.675,0.875,1]$} \\
\hline
\end{tabular}


Table 3 Coordinates of array of loudspeakers inside the enclosure along with the two-norm of secondary loudspeakers excitation voltage with and without coupling.

\begin{tabular}{|c|c|c|c|}
\hline Speaker & Position & $\begin{array}{c}\text { two-norm } \\
\text { (uncoupled) }\end{array}$ & $\begin{array}{c}\text { two-norm } \\
\text { (coupled) }\end{array}$ \\
\hline Primary speaker & {$[0.925,1,1.925]$} & - & - \\
\hline Secondary Speaker 1 & {$[0.5,0.925,1.8]$} & 3116.5 & 3192.4 \\
\hline Secondary Speaker 2 & {$[0.075,0.5,1.8]$} & 2235.4 & 2293.4 \\
\hline Secondary Speaker 3 & {$[0.925,0.5,1.8]$} & 2985.8 & 3063.6 \\
\hline Secondary Speaker 4 & {$[0.5,0.075,1.8]$} & 2208.5 & 2259.2 \\
\hline
\end{tabular}

associated maximum achievable potential energy reduction inside the enclosure are calculated using equations (51) and (52). Figure 10 shows the potential energy inside the enclosure, and the maximum potential energy reduction at the frequency range between 0 to $300 \mathrm{~Hz}$ when the exact potential energy, $E_{\mathrm{p}}$ in (45), and its approximation, $J_{\mathrm{p}}$ in (48), with and without coupling of secondary sources is minimized. Small deviation of the reduced potential energy in case of minimization of (45) and (48) indicates that the array of microphones shown in Fig 2(b) could capture most of the acoustic energy inside the enclosure. The simulations show the same amount of reduction in acoustic potential energy when the ASC system is active for both the coupled and uncoupled secondary sources. This is in agreement with part 1 of Theorem 2. However, differences between the two cases emerge when the magnitudes and phases of the excitation voltages applied to sources S1 to S4 are plotted in Fig. 11. As can be seen from this figure the energy of the applied voltages to all four loudspeakers in case of coupling is greater than the case when no coupling exists. This is shown in Fig. 12 by plotting the magnitude of the first and second parts of equation (60) against the frequency.

For typical parameters of a loudspeaker and acoustic properties of the enclosure, the values of $\left|Z_{s}\right|$ at each frequency are much larger than the magnitude of the frequency responses of the enclosure (elements of $\mathbf{Z}_{c}$ in (25)). Hence the magnitude of the second term at almost all frequencies is much larger than the first term. The exception is the case that the minimum eigenvalue of $\mathbf{Z}_{c}$ tends to zero. This can happen only at a few distinct frequencies (zeros of the frequency response of the system in the specified bandwidth), or when the ASC system is in the singularity condition as explained in Remark 2. 

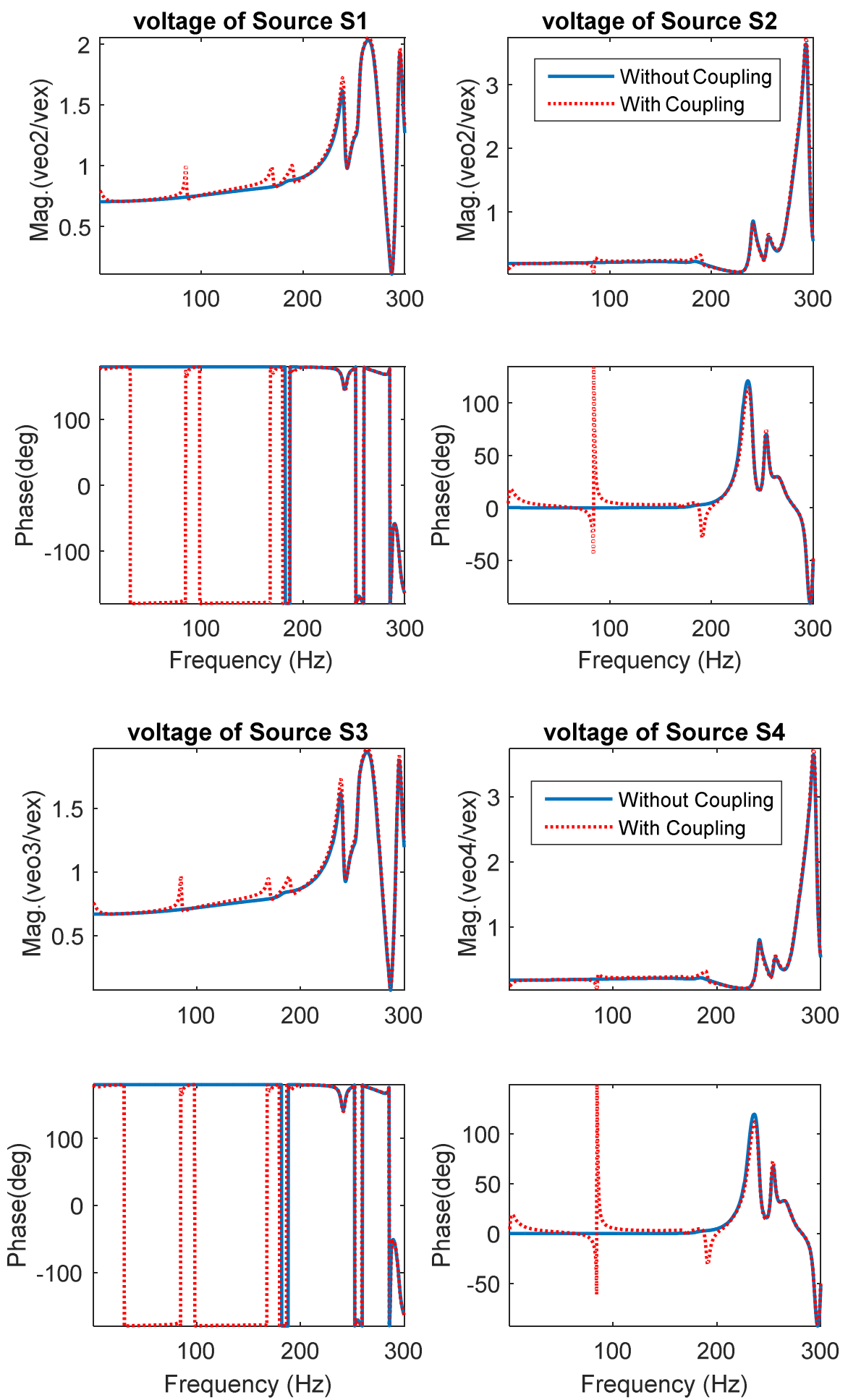

Figure 11. The magnitude and phase of optimal excitation voltages of S1, S2 (top), S3, and S4 (bottom) with and without coupling as a function of frequency. 


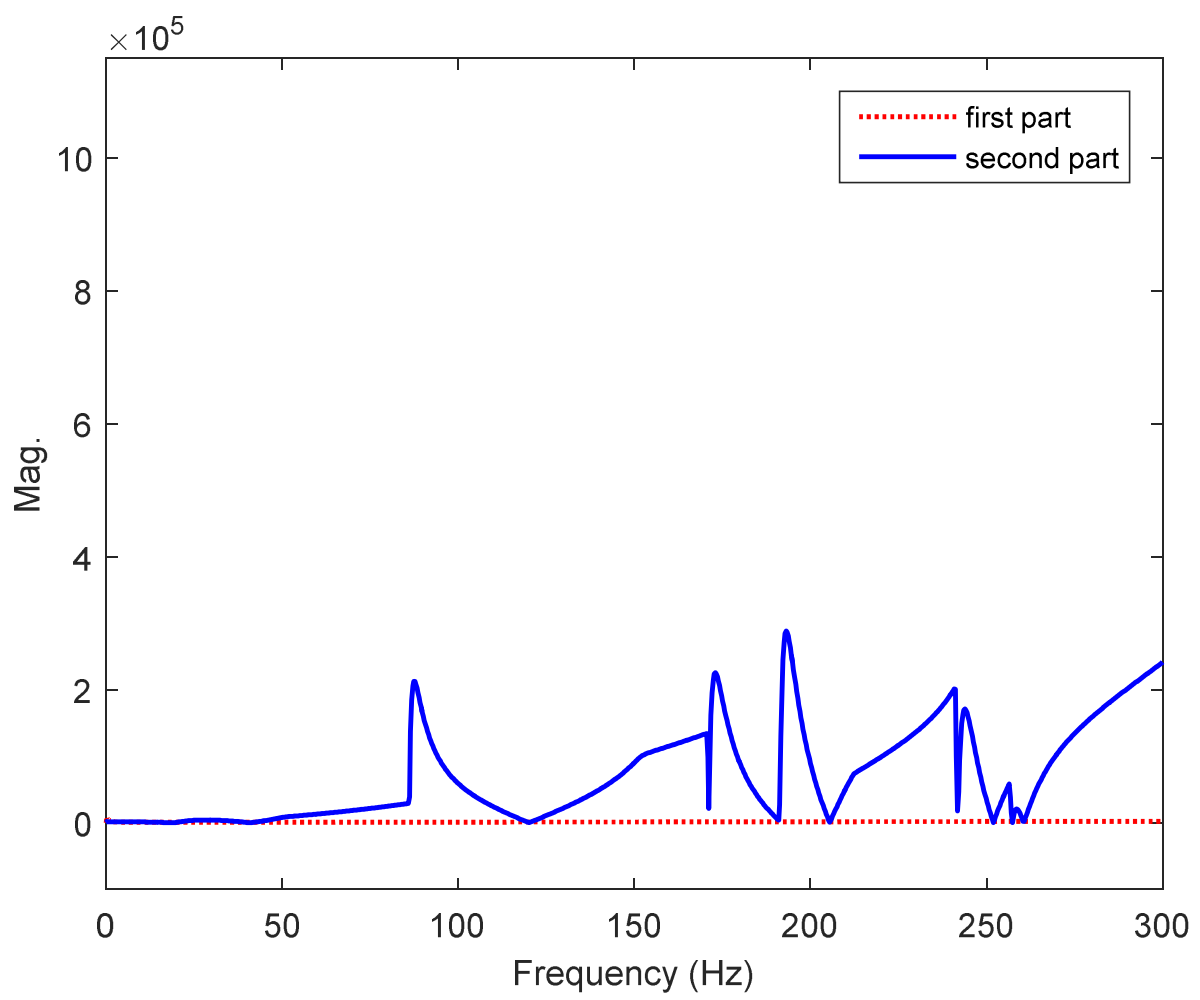

Figure 12. Magnitudes of the first and second parts of equation (60) as a function of frequency.

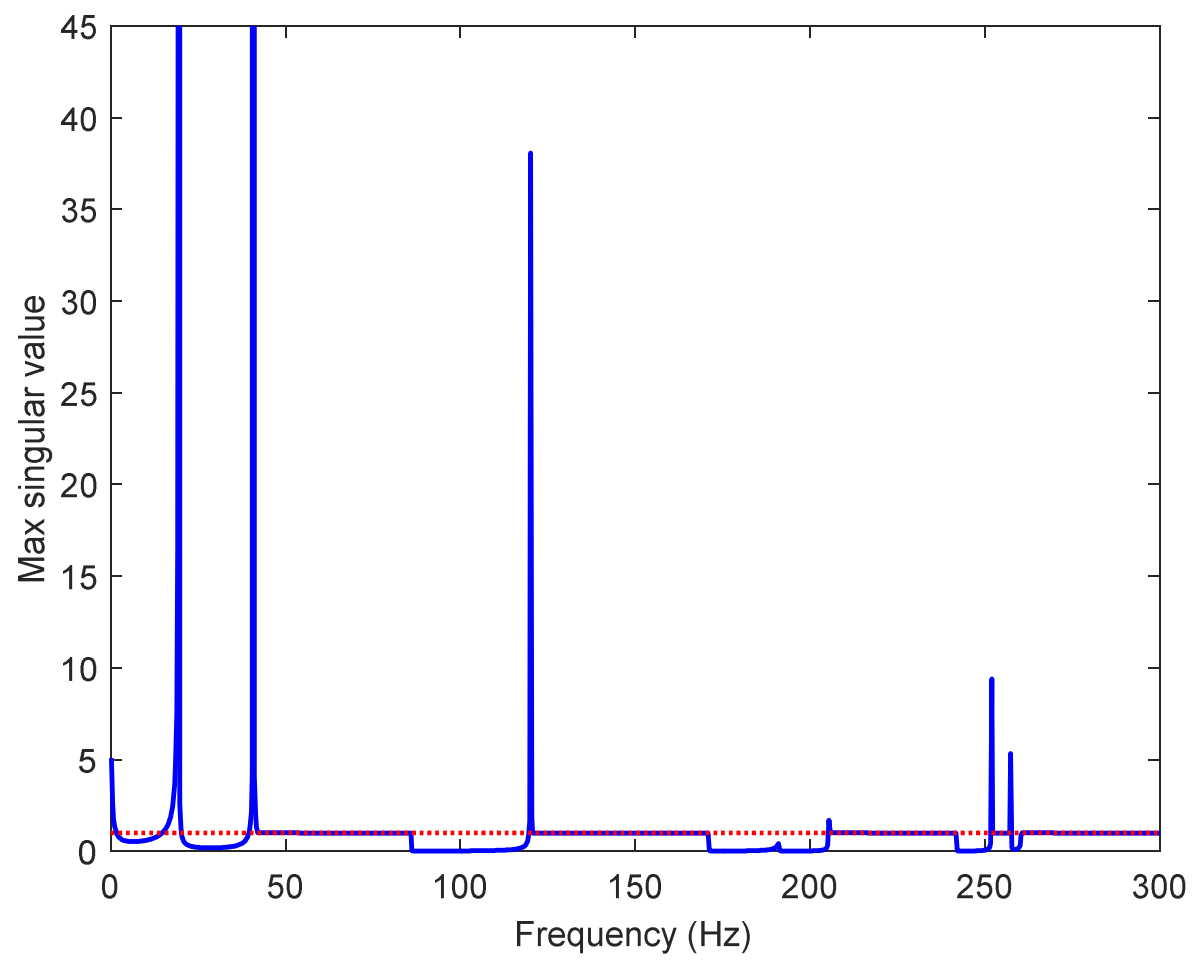

Figure 13. Maximum singular value of the coupling matrix $\overline{\mathbf{Z}}_{c}$ as a function of frequency. 
The maximum singular value of the coupling matrix $\overline{\mathbf{Z}}_{c}$ along with the separating line indicating ' 1 ' is plotted in Fig. 13. From these two figures it is clear that the maximum singular value of $\overline{\mathbf{Z}}_{c}$ is greater than one only at some distinct frequencies, which are the zeros of the frequency response of the system. In fact the zeros of the matrix $\mathbf{Z}_{c}$ occur at these frequencies, and as illustrated in Fig. 13, in the intervals between these frequencies the $\infty$-norm of $\overline{\mathbf{Z}}_{c}$ is less than one. The 2-norm of excitation voltages of all four sources with and without coupling is listed in Table 3. These results are in agreement with the statement and proof of the second part of Theorem 2 for the whole bandwidth of the system. The small peaks in the magnitude of the voltages applied to the loudspeakers correspond to the poles of the matrix $\mathbf{Z}_{c}$. At these frequencies $\bar{\sigma}\left[\overline{\mathbf{Z}}_{c}(\omega)\right]$ in (61) is smaller and hence its inverse causes greater voltage magnitude. Rapid changes in the phase of the excitation voltages in this figure are also due to the existence of these poles and zeros.

\section{CONCLUSIONS}

This article has considered the problem of modeling and analysis of coupling of secondary sources, with the aim of designing an active sound control system. In order to find an analytical closed form solution for the problem, at hand a rectangular enclosure with rigid boundary conditions is assumed to formulate the problem. For this purpose it is assumed that an array of $L$ loudspeakers and $M$ microphones are distributed inside the enclosure and the characteristic of the system are analyzed by looking at the frequency response functions of the system, as determined between the loudspeakers and microphones. The results summarized in several theorems and lemmas confirm that coupling of secondary loudspeakers will change the amplitude and phase of the optimal excitation voltage applied to the secondary sources, compared to the case when coupling of loudspeakers is not an issue. Furthermore, looking at the optimal value of the performance index, i.e. the potential energy inside the enclosure, reveals that coupling of secondary loudspeakers will not affect this value. This means the same control objective is achievable at the expense of more control effort by secondary loudspeakers. The analytical results are illustrated by means of numerical simulations to evaluate the performance of the active noise control system. Numerical simulations with two loudspeakers have been used to show the configurations that will lead to singularity and demonstrate the required strength of the secondary sources in comparison with a not coupled system. As the next step we will investigate how coupling of secondary loudspeakers will affect the performance of adaptive feedforward control 
algorithms for practical implementation of the system. In this regard, one approach would be to combine frequency domain subband adaptive algorithms with the model developed in this article.

\section{REFERENCES}

[1] Dimino, I., Aliabadi F., Active Control of Aircraft Cabin Noise: Computational and Experimental Methods in Structures, 2015, Imperial College Press, London, UK.

[2] Ross, C.F., Purver, M.R.J. “Active Cabin Noise Control,” in Proc. Of Active 97, 1997, Budapest, Hungry.

[3] Melchior F., Spors, S., "Spatial audio reproduction: from theory to production," in tutorial, 129th Convention of the AES, 2010, London, UK.

[4] Kolundzija, M., Faller, C., Vetterli, M., "Reproducing Sound Fields Using MIMO Acoustic Channel Inversion," Journal of Audio Engineering Society, 2011.

[5] Bullmore, A. J., Nelson, P. A., Curtis A. R. D., Elliott, S. J., "The Active Minimization of Harmonic Enclosed Sound Fields, Part II: A Computer Simulation,” Journal of Sound and Vibration, 1987, vol. 117, no. 1, pp. 15-33.

[6] Gauthier P.-A., Berry, A., "Sound-field reproduction in-room using optimal control techniques: Simulations in the frequency domain," Journal of Acoustic Society of America, vol. 117, no. 2, Feb. 2005.

[7] Elliott, S.J., Curtis, A.R.D., Bullmore, A.J., Nelson, P.A., "The Active Minimization of Harmonic Enclosed Sound Fields, Part III: Experimental Verification," Journal of Sound and Vibration, 1987, 117(1), pp. 35-58.

[8] Guo, J., Pan J., Hodgson, M., "Local or global control-the applicable active noise control strategy in enclosed environments," in Proceeding of Active 2002, 2002, ISVR, University of Southampton, UK.

[9] Hasheminejad M., Azarpeyvand M. "Modal Vibrations of an Infinite Cylinder in an Acoustic Halfspace," International Journal of Engineering Science, 2003, vol. 41, pp. 2253-2271.

[10] Lau, S.K., Tang, S.K., "Sound Fields in a Slightly Damped Rectangular Enclosure under Active Control," Journal of Sound and Vibration, 2000, vol. 238, no. 4, pp. 637-660.

[11] Maa D.Y., Sound Field in a Room and Its Active Noise Control. Applied Acoustics, 1994, vol. 41, pp. 113-126.

[12] Marx, D., Mao, X., Jaworski, A.J., “Acoustic Coupling Between the Loudspeaker and the Resonator in a Standing-Wave Thermoacoustic Device," Applied Acoustics, 2006, vol. 67, pp. 402-419.

[13] Montazeri, A., Poshtan, J., Kahaei M.H. "Optimal placement of loudspeakers and microphones in an enclosure using genetic algorithm," in proceeding of IEEE Conference on Control Applications, June 2003, vol. 1, pp. 135-139.

[14] Montazeri, A., Poshtan, J., Kahaei, M.H. "Analysis of the Global Reduction of Broadband Noise in a Telephone Kiosk Using a MIMO Modal ANC System," International Journal of Engineering Science, 2007, vol. 45, pp. 679-697.

[15] Montazeri, A., Poshtan, J., "GA-based optimization of a MIMO ANC system considering coupling of secondary sources in a telephone kiosk," Applied Acoustics, 2009, vol. 70, no. 7, pp. 945-953.

[16] Montazeri, A., Poshtan J., Poshtan, M. "Analysis of the Behavior of Coupled Loudspeakers in a MIMO ANC System in an Enclosure," IEEE International Conference on Control Applications (CCA), Sept. 2010, 228-233.

[17] Nelson, P.A., Curtis A.R.D., Elliott, S.J., Bullmore, A.J., "The Active Minimization of Harmonic Enclosed Sound Fields, Part I: Theory," Journal of Sound and Vibration, 1987, no. 117, vol. 1, pp. 113.

[18] Elliott, S.J., "Active noise and vibration control in vehicles," Vehicle Noise and Vibration Refinement, 2010, pp. 235-251.

[19] Sano, H., Yamashita, T., Nakamura, N., "Recent Application of Active Noise and Vibration Control to Automobiles," in Proc. Of Active 2002, 2002, Southampton, UK.

[20] Tseng, W.-K., Rafaely, B., Elliott, S.J., "Local Active Sound Control Using 2-Norm and $\infty$-Norm Pressure Minimization," Journal of Sound and Vibration, 2000, vol. 234, no. 3, pp. pp. 427-439.

[21] Zimmer, B.J., Lipshitz, S.P., Morris, K.A., Vanderkooy, J., Obasi, E.E., "An Improved Acoustic Model for Active Noise Control in a Duct," Journal of Dynamic Systems, Measurement, and Control, 2003, vol. 125, pp.382-395. 
[22] Cheer, J., Elliott, S.J., "Multichannel Control Systems for the Attenuation of Interior Road Noise in Vehicles," Mechanical Systems and Signal Processing, vol. 60-61, 2015, pp. 753 -769.

[23] Diaz, J., Egana, J.V., "A Local Active Noise Control System Based on a Virtual-Microphone Technique for Railway Sleeping Vehicle Applications," Mechanical Systems and Signal Processing, vol. 20, no. 8, 2006, pp. 2259-2276.

[24] Apostolopoulos, J.G., Chou, P.A., Culbertson, B., Kalker, T., Trott, M.D., Wee, S., "The Road to Immersive Communication," Proceedings of the IEEE, vol. 100, no. 4, pp. 974-990, April 2012.

[25] Huang, Y., Chen, J., Benesty, J. , "Immersive Audio Schemes," IEEE Signal Processing Magazine, vol. 28, no. 1, pp. $20-32,2011$.

[26] M. Vidyasagar, Control systems synthesis: a factorization approach. The MIT Press, Cambridge, MA, USA, 1985.

[27] Montazeri, A., Poshtan, J., Kahaei, M.H., "Optimal placement of loudspeakers and microphones in an enclosure using genetic algorithm," in Proceedings of 2003 IEEE Conference on Control Applications, vol. 1, pp. 135-139, 2003.

[28] Montazeri, A., Poshtan, J., "Optimizing a multi-channel ANC system for broadband noise cancellation in a telephone kiosk using genetic algorithms," Shock and Vibration, vol. 16, no. 3, pp. 241-260, 2009.

[29] Montazeri, A., Poshtan, J., "GA-based optimization of a MIMO ANC system considering coupling of secondary sources in a telephone kiosk," Applied Acoustics, vol. 70, No. 7, pp. 945-953, 2009.

[30] Montazeri, A., Poshtan, J., Yousefi-Koma, A., "The use of particle swarm to optimize the control system in a PZT laminated plate, Smart Materials and Structures, vol. 17, no. 4, 045027, 2008.

[31] Chen, C.T., Linear System Theory and Design. New York: Holt, Rinehart and Winston, 1984.

[32] Salgado M.E., Conley A., "MIMO interaction measure and controller structure selection," International Journal of Control, vol. 77, no. 4, pp. 367-383, 2007. 